\title{
The integrated stress response mediates necrosis in murine Mycobacterium tuberculosis granulomas
}

\author{
Bidisha Bhattacharya, ${ }^{1}$ Shiqi Xiao, ${ }^{2}$ Sujoy Chatterjee, ${ }^{1}$ Michael Urbanowski, ${ }^{2}$ Alvaro Ordonez, ${ }^{2}$ Elizabeth A. Ihms, ${ }^{2}$ \\ Garima Agrahari, ${ }^{1}$ Shichun Lun, ${ }^{2}$ Robert Berland, ${ }^{1,3}$ Alexander Pichugin, ${ }^{4}$ Yuanwei Gao, ${ }^{5}$ John Connor, ${ }^{6}$ Alexander R. Ivanov, ${ }^{7}$ \\ Bo-Shiun Yan, ${ }^{8}$ Lester Kobzik, ${ }^{9}$ Bang-Bon Koo, ${ }^{1}$ Sanjay Jain, ${ }^{2}$ William Bishai, ${ }^{2}$ and Igor Kramnik ${ }^{1,10}$

\begin{abstract}
${ }^{1}$ The National Emerging Infectious Diseases Laboratory, Boston University School of Medicine, Boston, Massachusetts, USA. ${ }^{2}$ Center for TB Research, Johns Hopkins University School of Medicine, Baltimore, Maryland, USA. ${ }^{3}$ Department of Integrative Physiology and Pathobiology, Tufts University School of Medicine, Boston, Massachusetts, USA. ${ }^{4}$ Department of Cellular Immunology, Walter Reed Army Institute of Research, Silver Spring, Maryland, USA. 5Department of Pharmacokinetics, Pharmacodynamics \& Drug Metabolism (PPDM), Merck, West Point, Pennsylvania, USA. ${ }^{6}$ Department of Microbiology, Boston University School of Medicine, Boston, Massachusetts, USA. Department of Chemistry \& Chemical Biology, Northeastern University, Boston, Massachusetts, USA. ${ }^{8}$ Institute of Biochemistry and Molecular Biology, National Taiwan University Medical College, Zhongzheng District, Taipei City, Taiwan. ${ }^{9}$ Harvard T.H. Chan School of Public Health, Boston, Massachusetts, USA. ${ }^{10}$ Department of Medicine, Pulmonary Center, Boston University School of Medicine, Boston, Massachusetts, USA.
\end{abstract}

\begin{abstract}
The mechanism by which only some individuals infected with Mycobacterium tuberculosis develop necrotic granulomas with progressive disease while others form controlled granulomas that contain the infection remains poorly defined. Mice carrying the sst1-suscepible $\left(s s t 1^{5}\right)$ genotype develop necrotic inflammatory lung lesions, similar to human tuberculosis (TB) granulomas, which are linked to macrophage dysfunction, while their congenic counterpart (B6) mice do not. In this study we report that (a) sst $1^{5}$ macrophages developed aberrant, biphasic responses to TNF characterized by superinduction of stress and type I interferon pathways after prolonged TNF stimulation; (b) the late-stage TNF response was driven via a JNK/IFN- $\beta$ / protein kinase R (PKR) circuit; and (c) induced the integrated stress response (ISR) via PKR-mediated elF2 $\alpha$ phosphorylation and the subsequent hyperinduction of ATF3 and ISR-target genes Chac1, Trib3, and Ddit4. The administration of ISRIB, a small-molecule inhibitor of the ISR, blocked the development of necrosis in lung granulomas of $M$. tuberculosis-infected sst $1^{5}$ mice and concomitantly reduced the bacterial burden. Hence, induction of the ISR and the locked-in state of escalating stress driven by the type I IFN pathway in $s s t 1^{5}$ macrophages play a causal role in the development of necrosis in TB granulomas. Interruption of the aberrant stress response with inhibitors such as ISRIB may offer novel host-directed therapy strategies.
\end{abstract}

\section{Introduction}

Understanding mechanisms driving necrotization of tuberculosis (TB) lesions may offer a promising path toward effective hostdirected therapies. The formation of the TB granuloma is a core virulence mechanism that enables pathogen survival, sanctuary from immune clearance mechanisms, and transmission (1), and the trajectories of TB granuloma progression vary among infected humans and in nonhuman primate models (2). Recent studies demonstrated roles of Mycobacterium tuberculosis-secreted necrotizing toxin, as well as excess free iron and ferroptosis in macrophage death and the formation of necrotic TB lesions, respectively $(3,4)$. However, why certain individuals develop necrotic granulomas and progress to cavitation and others do not remains unclear.

It has long been known that granuloma formation requires TNF, a cytokine essential for host resistance to TB both in humans and in animal models (5). However, recent studies demonstrated a role of elevated TNF levels in TB immunopathology $(6,7)$.

Authorship note: BB and SX contributed equally to this work. Conflict of interest: The authors have declared that no conflict of interest exists. Copyright: @ 2021, American Society for Clinical Investigation. Submitted: May 17, 2019; Accepted: December 4, 2020; Published: February 1, 2021 Reference information: J Clin Invest. 2021;131(3):e130319. https://doi.org/10.1172/JCl130319.
Hyperactivation of the type I IFN (IFN-I) pathway has been clearly associated with TB progression in human patients (8), as well as in animal models (9). In those studies, both higher virulence of $M$. tuberculosis strains and greater host susceptibility to M. tuberculosis were associated with increased expression of IFN-I-stimulated genes (ISGs), while blockade of the IFN-I pathway in mouse models increased host resistance. The upregulation of ISG expression in peripheral blood was detected in individuals with latent TB infection up to 18 months before clinical diagnosis of TB disease, indicating a causal role of the IFN-I pathway activation in TB progression in humans $(10,11)$. Among IFN-mediated mechanisms of TB susceptibility, the induction of soluble mediators, such as IL-10 and IL-1 receptor antagonist (IL-1Ra) that suppress essential effector pathways of anti-TB immunity, has been demonstrated using mutant and genetically engineered mouse models $(9,12)$. However, mechanisms for how this cytokine milieu emerges within TB lesions and whether it leads to formation of necrotic TB granulomas in TB-susceptible hosts remain unknown.

An important tool for the study of necrosis within TB lesions is the $\mathrm{C} 3 \mathrm{HeB} / \mathrm{FeJ}$ mouse, which develops necrotic TB lesions and even cavities in the lungs after infection with both laboratory strains and clinical isolates of virulent $M$. tuberculosis (13). It has been extensively validated and adopted for use in preclinical studies of TB drugs and vaccines (14). In this mouse strain, the sst 1 locus 
was found to specifically control the necrotization of TB granulomas in the lungs $(15,16)$. B6 mice carrying the sst1-suscepible $\left(s s t 1^{S}\right)$ genotype (B6.Sst1 ${ }^{\mathrm{S}}$ mice) carry the $\mathrm{C} 3 \mathrm{HeB} / \mathrm{FeJ}$-derived $s s t 1$ locus on a B6 background and develop well-organized necrotic TB granulomas in the lungs (17). Although the B6.Sst1 ${ }^{\mathrm{s}}$ mice initially control M. tuberculosis replication similarly to their parental B6 mice, the necrotic TB granulomas emerge within 8-12 weeks after aerosol infection (17). Remarkably, necrotic granuloma formation is observed only in the lungs of the B6.Sst $1^{\mathrm{S}}$ mice, while granulomas elsewhere do not progress toward necrosis. Therefore, comparing sst1-congenic B6.Sst1 ${ }^{\mathrm{s}}$ with parental B6 (WT) mice that do not develop necrotic TB granulomas provides a valuable tool for studying pathways that lead to necrosis.

The sst1 locus has been found to encode the SP100 family of nuclear proteins (15). The mouse Sp110/Ipr1 gene is not expressed in the mutant $\mathrm{B} 6 . \mathrm{Sst}^{\mathrm{S}}$ macrophages. Its human homolog has been shown to control macrophage activation and susceptibility to TB in humans (18). Another gene encoded in the mouse sst 1 locus and missing in the B6.Sst1 ${ }^{\mathrm{s}}$ mice, SP140, has been associated in human genome-wide association studies (GWAS) with susceptibility to chronic autoimmune and inflammatory diseases including Crohn's disease and multiple sclerosis (19). In dogs, a closely related family member, SP110, was associated with amyotrophic lateral sclerosis (ALS) (20). Thus, the SP100 family members encoded in the sst 1 locus, which are induced in activated WT B6 macrophages and not expressed in activated B6.Sst $1^{\mathrm{S}}$ macrophages, are clearly implicated in aberrant inflammatory and neurodegenerative diseases in several species including humans (reviewed in ref. 21).

Previously, we have shown that the sst1-mediated susceptibility does not lead to systemic immunodeficiency and that $\mathrm{T}$ cell function is not compromised in $s s t 1^{S}$ mice. Conversely, the sst1-mediated phenotype has been attributed to bone marrowderived myeloid cells and associated with hyperactivity of the IFN-I pathway $(15,22)$. Indeed, in a recent study B6.Sst1 ${ }^{\mathrm{S}}$ mice exhibited marked elevations of IFN- $\beta$ in the lungs upon $M$. tuberculosis infection (23). Moreover, infected $\mathrm{IFNAR}^{-/}$mice in the B6.Sst $1^{\text {s }}$ background demonstrated reduced immunopathology compared with B6.Sst $1^{\mathrm{S}}$ mice, thus establishing that progression of pulmonary necrosis in the $M$. tuberculosis-infected host requires the IFN-I pathway. This IFN-I response was essential for loss of containment via induction of IL-1Ra, and heterozygous deficiency in the IL-1Ra was found to be protective. These results reveal a distal IFN-I-driven immunologic signaling mechanism of TB susceptibility via dysregulation of a cytokine network.

We sought to understand the basis for the increased level of IFN-I signaling and its impact on necrosis. We found that stimulation of B6.Sst ${ }^{\mathrm{S}}$ macrophages with TNF induced an aberrant response characterized by a late-phase superinduction of the IFN-I pathway. It required persistent stimulation with TNF and was driven by reactive oxygen species (ROS), stress kinases, and proteotoxic stress. Transcriptomic analysis revealed aberrant triggering of the integrated stress response (ISR) - a metabolic safety valve mechanism leading to reduced protein synthesis and increased expression of repair and biochemical readjustment pathways in severely stressed cells (24). Very interestingly, ISR markers have already been found to be strongly upregulated in human necrotic granulomas specifically in cellular layers close to necrotic centers of the TB granulomas (25). We found that the deleterious ISR was driven by TNF via IFN-I-mediated induction of the eIF $2 \alpha$ kinase, protein kinase R (PKR). Confirming the in vivo relevance of these findings, upon treatment of B6.Sst ${ }^{\mathrm{S}}$ mice with a small-molecule inhibitor of the ISR known as ISRIB, we observed inhibition of granuloma necrosis and a concomitant reduction in bacterial CFU counts. These findings demonstrate a role of IFN- $\beta$ mediated ISR as an emergency regulator of macrophage proteostasis during chronic inflammation and reveal a noncanonical metabolic pathway that leads to necrosis in TB granulomas that is targetable with host-directed therapy.

\section{Results}

TNF triggers hyperactivity of IFN-I and stress pathways in B6.Sst $1^{S}$ macrophages. To begin dissecting mechanisms behind the upregulated IFN- $\beta$ production, we compared IFN- $\beta$ secretion by the WT B6 and B6.Sst1 ${ }^{\mathrm{S}}$ bone marrow-derived macrophages (BMDMs), stimulated either with TNF (which induces low levels of IFN- $\beta$ in B6 macrophages, ref. 26) or the classical IFN- $\beta$ inducer poly(I:C). The B6.Sst $1^{\mathrm{S}}$ macrophages secreted higher levels of IFN- $\beta$ protein in response to both stimuli (Figure 1A and Supplemental Figure 1A; supplemental material available online with this article; https://doi.org/10.1172/JCI130319DS1). Next, we compared the kinetics of TNF-induced IFN- $\beta$ mRNA expression in WT B6 versus B6.Sst ${ }^{\mathrm{S}}$ BMDMs. Initially, TNF induced similarly low levels of IFN- $\beta$ mRNA expression in both cell types. Then, while IFN- $\beta$ levels remained relatively stable in WT B6 macrophages, in the B6.Sst ${ }^{\mathrm{S}}$ cells the IFN- $\beta$ mRNA expression significantly increased between 8 and 24 hours, such that the strain difference in IFN- $\beta$ mRNA levels reached 10- to 20 -fold by 24 hours (Figure 1B). In addition, the B6.Sst $1^{\mathrm{s}}$ macrophages stimulated with TNF expressed significantly higher levels of the IFN-stimulated gene Rsad2 (viperin, Supplemental Figure 1B), whose upregulation we found was reduced by $50 \%-75 \%$ in the presence of IFN type I receptor-blocking (IFNAR1-blocking) antibodies (E. Brownhill, personal communication), thus confirming hyperactivation of the IFN-I pathway in the B6.Sst ${ }^{\mathrm{s}}$ cells. The IFN- $\beta$ and Rsad 2 mRNA expression kinetics demonstrated that the bias toward IFN-I pathway activation in the B6.Sst $1^{\mathrm{s}}$ macrophages occurred at a later stage of persistent stimulation with TNF.

To characterize effects of the sst 1 locus on the late response of primary macrophages to TNF more broadly, we compared transcriptomes of WT B6 and B6.Sst $1^{\mathrm{S}}$ BMDMs after 18 hours of stimulation with TNF at $10 \mathrm{ng} / \mathrm{mL}$ (Figure 1C). Although no significant differences were detected in naive macrophages, the gene expression profiles of TNF-treated cells diverged substantially, with 592 genes significantly differentially expressed $(P<0.001 ;$ Supplemental Table 1). Those included the Sp110/ Ipr1 and Sp140 mRNAs encoded within the sst1 locus that were expressed and upregulated by TNF only in the WT B6 BMDMs. The most prominent differentially expressed gene cluster was composed of genes that were selectively upregulated by TNF in B6.Sst1 ${ }^{\text {s }}$, but not WT B6 macrophages (Figure 1C). Using gene set enrichment analysis (GSEA) we found significant enrichment for the IFN-I-regulated genes in B6.Sst $1^{\mathrm{s}}$ macrophages responding to TNF. Genes involved in nuclear RNA processing and nuclear- 
A

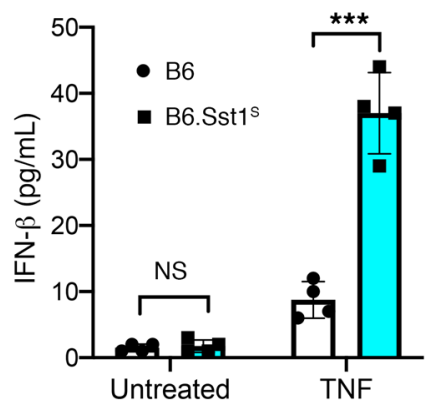

B

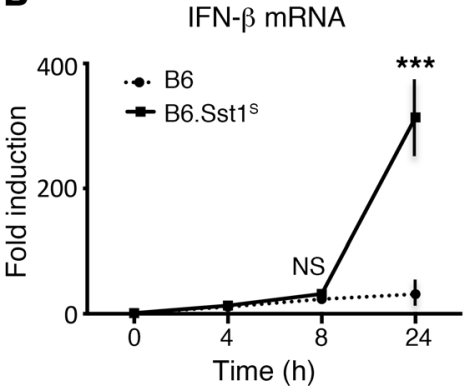

D

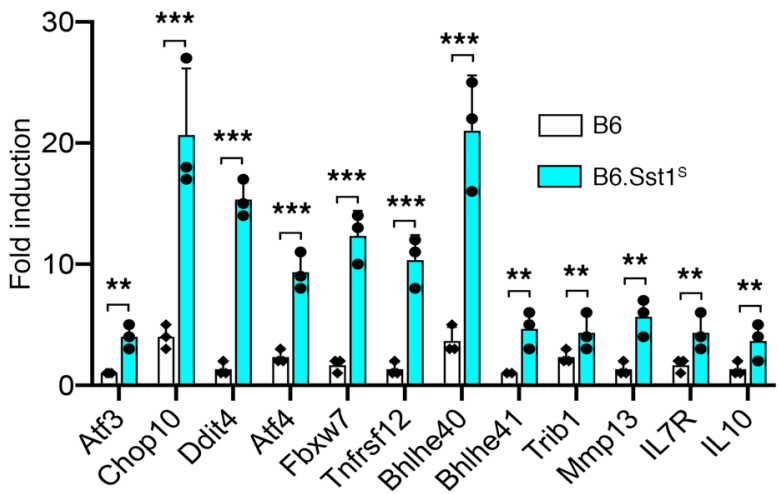

C

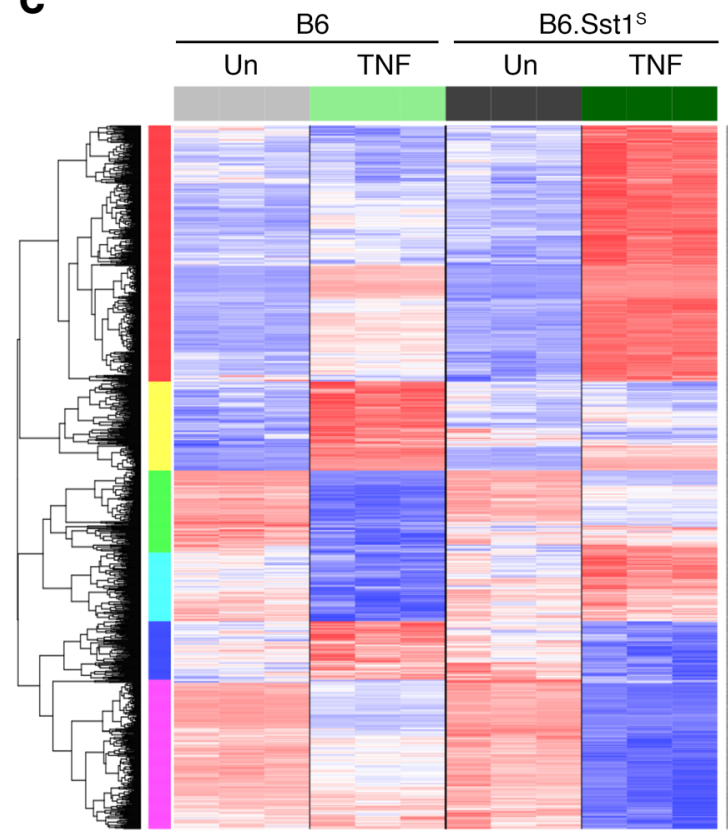

E

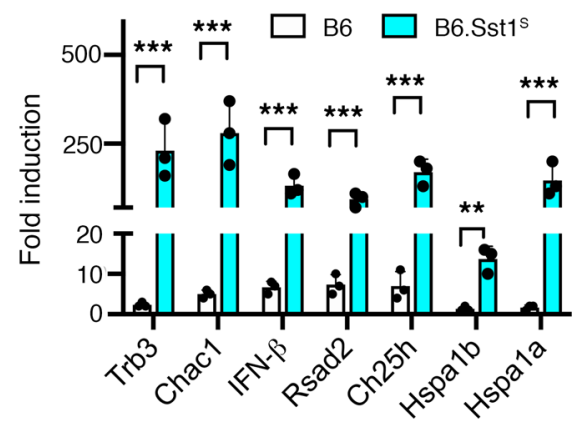

Figure 1. Superinduction of IFN- $\beta$ in B6.Sst $1^{5}$ BMDMs after prolonged stimulation with TNF. (A) IFN- $\beta$ protein concentration in supernatants of WT B6 and B6.Sst $1^{5}$ BMDMs treated with $10 \mathrm{ng} / \mathrm{mL}$ TNF- $\alpha$ for 24 hours was detected using ELISA. Results represent data from 2 independent experiments. (B) Time course of IFN- $\beta$ mRNA expression in B6.Sst $1^{5}$ and WT B6 BMDMs after treatment with $10 \mathrm{ng} / \mathrm{mL}$ TNF, as determined using qRT-PCR. The data are representative of 3 biological replicates. (C) Comparison of gene expression profiles of B6.Sst1 ${ }^{\mathrm{s}}$ versus WT B6 BMDMs stimulated with TNF (10 ng/mL) or unstimulated (un) for 18 hours using hierarchical clustering. The global gene expression was determined using Affymetrix GeneChip Mouse Gene 2.0 Arrays. (D) Validation of microarray data using gene-specific qRT-PCR. (E) Differential stress response and IFN-I pathway gene expression in TNF-stimulated B6.Sst ${ }^{\mathrm{S}}$ and WT B6 BMDMs. Data are representative of at least 3 independent experiments. In panels B, D, and E, the qRT-PCR data were normalized to 185 rRNA and are presented relative to the expression in untreated B6 cells (set to 1 ). ${ }^{* *} P \leq 0.01 ;{ }^{* *} P \leq 0.001$ by Welch's $t$ test. NS, not significant.

cytoplasmic transport were also upregulated by TNF in the B6.Sst ${ }^{\text {s }}$ BMDMs. Strikingly, multiple biosynthetic pathways were coordinately downregulated in TNF-stimulated B6.Sst $1^{\mathrm{s}}$ macrophages, including lipid and cholesterol biosynthesis, protein translation, ribosome, mitochondrial function, and oxidative phosphorylation (Supplemental Table 2). Further validation of the differential gene expression using quantitative real-time reverse transcription PCR (qRT-PCR) demonstrated upregulation of a number of other genes important for pathogenesis such as IL-10, Mmp-13, IL-7R, death receptor 3 (Dr3/Tnfrsf12), and transcription factors Bhlhe 40 and Bhlhe 41 in the B6.Sst $1^{\mathrm{s}}$ cells (Figure 1D). Significant upregulation of IFN- $\beta$ and the typical IFN-I-inducible genes Rsad2 and Ch25h confirmed our previous observations of IFN-I hyperactivity. Also, a group of genes (Atf3, Chop10, Ddit4, Trib3, and Chac1) induced during the ISR, as well as genes (Hspa1a and Hspa1b) known to be markers of proteotoxic stress (PS), were significantly enriched among the upregulated genes, and their upregulation by TNF specifically in B6.Sst ${ }^{\mathrm{s}}$ macrophages was confirmed using qRT-PCR (Figure 1E).

Prolonged TNF stimulation induces biphasic progression of the ISR and proteome remodeling in B6.Sst $1^{S}$ macrophages. Upon TNF stimulation, the Trib3 and Chac1 genes were among the most highly upregulated late-response genes in the B6.Sst $1^{\mathrm{s}}$ BMDMs. These genes are known targets of the transcription factor Chop10 (Ddit3), which is activated downstream of the ISR transcription factors ATF4 and ATF3. Collectively, this pathway 
A
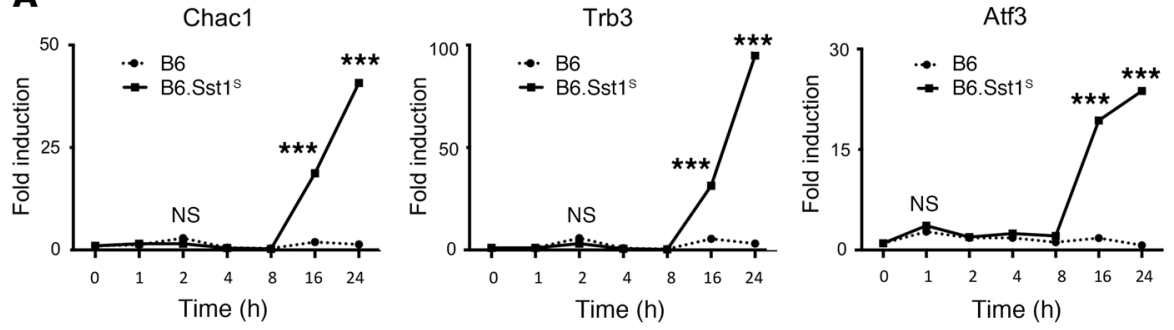

B
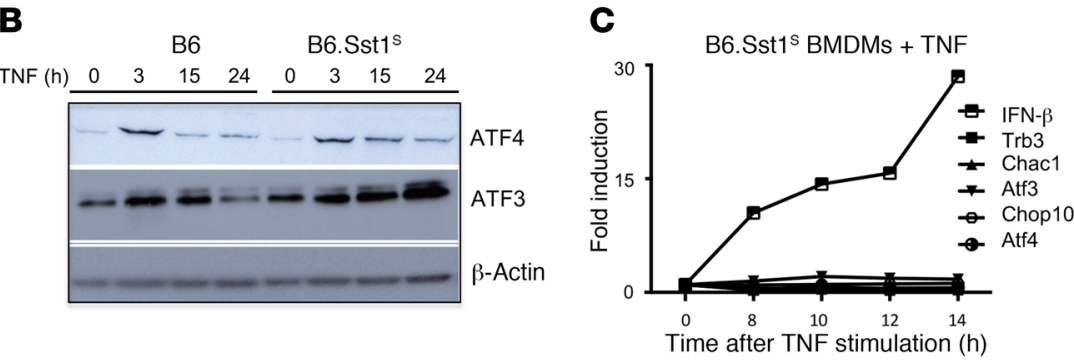

D

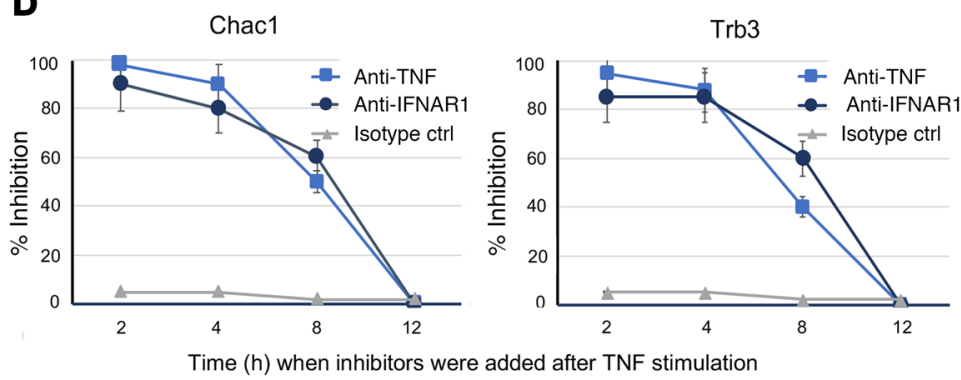

$\mathbf{E}$

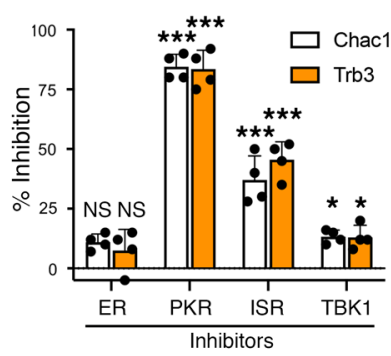

F

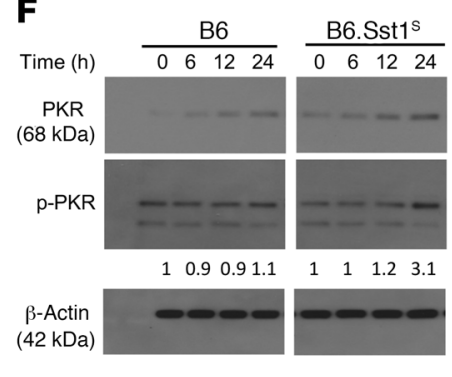

Figure 2. TNF treatment leads to biphasic upregulation of the ISR in B6.Sst $1^{5}$ BMDMs.

(A) Kinetics of integrated stress response (ISR) gene expression in $\mathrm{B} 6.5 \mathrm{st} 1^{\mathrm{S}}$ and $\mathrm{WT} \mathrm{B} 6 \mathrm{BMDMs}$ treated with TNF $(10 \mathrm{ng} / \mathrm{mL})$. (B) Kinetics of ISR proteins in TNF-stimulated WT B6 and B6.Sst $1^{5}$ BMDMs (representative of 2 biological replicates). (C) Kinetics of IFN- $\beta$ and ISR gene expression in B6.Sst $1^{5}$ BMDMs 8-14 hours after stimulation with TNF. (D) Effects of TNF and IFNAR1 blockade on Chac1 and Trb3 mRNA expression in B6.Sst $1^{5}$ BMDMs stimulated with TNF. The neutralizing anti-IFNAR1 and antiTNF- $\alpha$ or isotype control antibodies were added 2-12 hours after TNF. The mRNA expression was measured at 16 hours of TNF stimulation and percentage inhibition by the neutralizing antibodies added at each time point was calculated with respect to cells treated with TNF alone. (E) Effect of the ER stress (PBA), PKR (C16), ISR (ISRIB), and TBK1 (BX795) inhibitors on latephase ISR gene expression in TNF-stimulated B6.Sst $1^{5}$ BMDMs. The inhibitors were added 12 hours after TNF stimulation, and the mRNA levels were measured at 16 hours. (F) Kinetics of PKR protein expression and phosphorylation (p-PKR) in B6.5st1 ${ }^{\mathrm{s}}$ and WT B6 BMDMs treated with TNF. Ratios of $p$-PKR to those in unstimulated BMDMs are presented for each time point. The Western blot is representative of 2 independent experiments. In panels $\mathbf{A}$ and C-E, 2-way ANOVA with Tukey's post hoc test was used on combined data of 3 independent experiments $\left({ }^{*} P=0.01-0.05 ;{ }^{* *} P<0.001\right)$. The qRT-PCR data were normalized to 185 rRNA and are presented in panels $\mathbf{A}$ and $\mathbf{C}$ relative to expression in untreated cells (set to 1 ). Percentage inhibition in panels $\mathbf{D}$ and $\mathbf{E}$ was calculated as compared to fold induction by TNF in the absence of inhibitors. NS, not significant. is known as the ISR (27). Comparing the mRNA kinetics of genes representing transcriptional targets of the ISR (Atf3, Chop10, Chac1, Trb3, and Ddit4) during the course of TNF stimulation, we observed that the expression of the ISR genes spiked in the B6.Sst1 ${ }^{\mathrm{S}}$ cells at 16 hours and continued to increase further between 16 and 24 hours (Figure 2A). Next, we monitored the expression of ISR markers ATF4 and ATF3 at the protein level by Western blot. Initially, we observed similar induction of ATF4 and ATF3 after 3 hours of TNF stimulation in both the WT B6 and B6.Sst1 ${ }^{\mathrm{S}}$ BMDMs. However, in the WT B6 cells the levels of ATF4 and ATF3 proteins decreased to basal levels by 15 and 24 hours, respectively. In contrast, the ATF3 levels increased in the B6.Sst1 ${ }^{\mathrm{S}}$ macrophages during the 15- to 24-hour interval (Figure $2 \mathrm{~B})$. Thus, the $s s t 1^{S}$ allele is uniquely associated with escalated transcription and translation of ISR genes after 12 hours of TNF stimulation, the timing of which closely resembles the kinetics of the IFN-I pathway induction by TNF in B6.Sst1's macrophages.
We followed the kinetics of the ISR- and IFN-inducible genes within a critical period between 8 and 14 hours at 2-hour intervals. The IFN- $\beta$ mRNA expression level in the B6.Sst1 ${ }^{\mathrm{s}}$ macrophages gradually increased, while the ISR markers remained at the same level throughout this period, suggesting a possible mechanistic hierarchy (Figure 2C). Therefore, we tested whether blocking IFN-I signaling reduced the ISR induction. IFNAR1-blocking antibodies were added at different times after stimulation with TNF (10 ng/mL), and the ISR was assessed at 16 hours following TNF stimulation, as measured by Chac1 and Trb3 gene expression (Figure 2D). Blocking the IFN-I and TNF signaling using neutralizing antibodies at 2-4 hours after TNF stimulation profoundly suppressed the ISR escalation. However, the TNF and IFN-I blockade was only partially efficient at 8 hours and completely disappeared by 12 hours of TNF stimulation (Figure 2D). Thus, the ISR pathway was set in motion and initiated by TNF in an IFN-I-dependent manner, but its transition from the latent to 

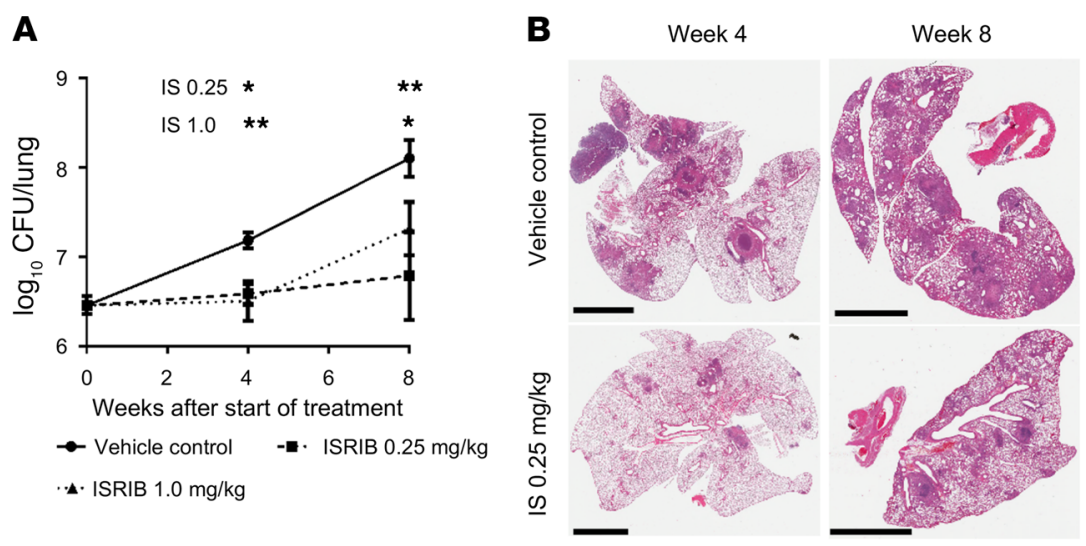

C
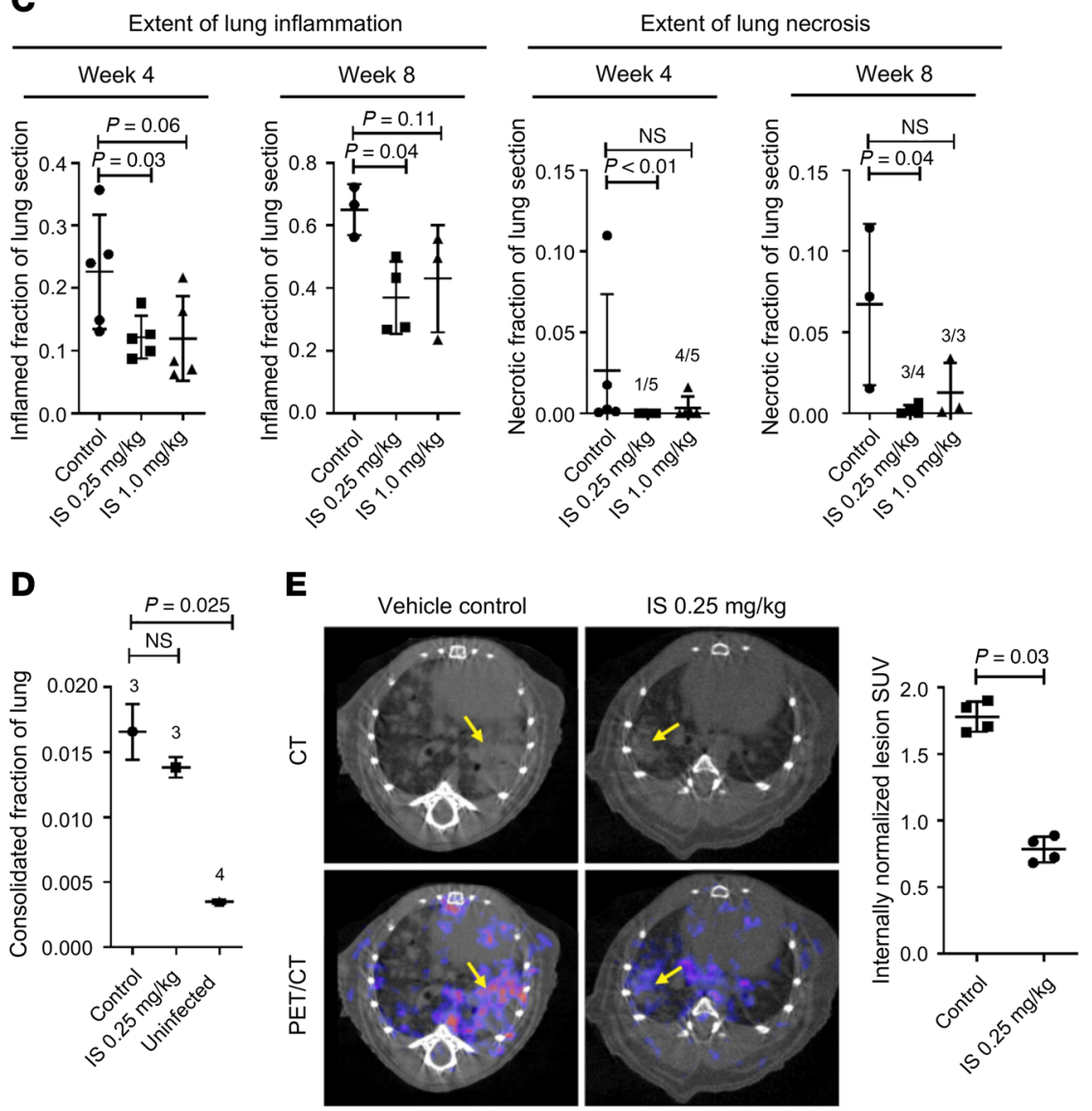

Figure 3. Effect of ISR inhibitor ISRIB on tuberculosis progression in B6.5st $1^{5}$ mice. For all figure panels, IS = ISRIB. (A) Lung bacillary loads at weeks 4 and 8 after treatment start. Data plotted as means \pm SEM. Asterisks indicate significant differences between treatment groups compared with vehicle control group calculated by 2-way ANOVA. *Indicates significance at $90 \% \mathrm{Cl} .{ }^{* *}$ Indicates significance at $95 \% \mathrm{Cl}$. (B) Representative $\mathrm{H} \& \mathrm{E}$-stained lung tissue slices from mice in the vehicle control group and the ISRIB-treated group with $0.25 \mathrm{mg} / \mathrm{kg}$ by body weight. Scale bars: $3 \mathrm{~mm}$. (C) Extent of inflammation and necrosis in lungs of mice treated with vehicle (control) or ISRIB. Each data point represents a survey of 1 mouse. All $P$ values were calculated based on a nonparametric Kruskal-Wallis test comparing the control to each of the dosing groups while accounting for multiple comparisons. NS, $P>0.20$. Fractions above group columns indicate the numbers of mice where necrosis was identified divided by the number of mice in the analysis group. (D) Fraction of lung volume with disease density as a quantitative measure of severity of disease in mouse lungs at week 8 . Data summary elements represent mean fraction of voxels \pm SEM. $P$ values calculated based on Welch's $t$ test. NS, not significant. Numbers above group columns indicate number of mice surveyed. (E) $\left[{ }^{18} \mathrm{~F}\right] \mathrm{FMISO} \mathrm{PET} / \mathrm{CT}$ imaging of mouse lungs at week 8. Quantification is given as doseand decay-corrected standardized uptake value (SUV) of lesions normalized to the PET signal from PET-blinded CT-scan selection of nondisease lung space volumes. $P$ values calculated by nonparametric 2-tailed Mann-Whitney test. For graphs in $\mathbf{C}$ and $\mathbf{E}$, data summary elements indicate means \pm SD. overt ISR in the B6.Sst1 ${ }^{\mathrm{S}}$ macrophages after 12 hours of TNF stimulation was TNF and IFN independent. Therefore, we searched for the drivers of the ISR during this transition.

$P K R$ is required for triggering the late ISR in the B6.Sst $1^{S}$ background. The ISR is known to be induced as a result of the inhibition of cap-dependent translation mediated by phospho-eIF $2 \alpha$ after phosphorylation by protein kinases activated in response to various stresses: viral infection and double-stranded RNA (dsRNA) (PKR), ER stress (PERK), starvation (GCN2), oxidative stress, and hypoxia (HIPK/HRI, ref. 28). To elucidate the driving force behind the late ISR transition in the $\mathrm{B} 6 . \mathrm{Sst}^{\mathrm{S}}$ macrophages, we measured the post-TNF induction of Trb3 and Chac1 mRNAs in B6.Sst1 ${ }^{\mathrm{S}}$ BMDMs in the presence of small molecules that inhibit the ISR (ISRIB, an eIF2 $\alpha$ phosphorylation inhibitor; ref. 29), ER stress (PBA, ref. 30), PKR (C16, ref. 31), and JNK (SP600125) (Figure 2E). Added at 12 hours after TNF stimulation, the ISR and PKR inhibitors (ISRIB and C16, respectively) profoundly inhibited the upregulation of both sentinel mRNAs, while the ER stress inhibitor PBA had no effect. This suggests that PKR activity was responsible for the transition from latent to overt ISR specifically in the B6.Sst1 ${ }^{\mathrm{S}}$ macrophages. Indeed, while PKR levels increased modestly in both WT B6 and B6.Sst $1^{\mathrm{s}}$ macrophages following TNF stimulation, only the B6.Sst $1^{\mathrm{s}}$ macrophages displayed significant late increases in the levels of active, phosphorylated PKR: $30 \%$ at 12 hours and $110 \%$ above baseline at 24 hours (Figure $2 \mathrm{~F}$ ). PKR is a classical IFN-inducible protein whose kinase activity is induced 
A

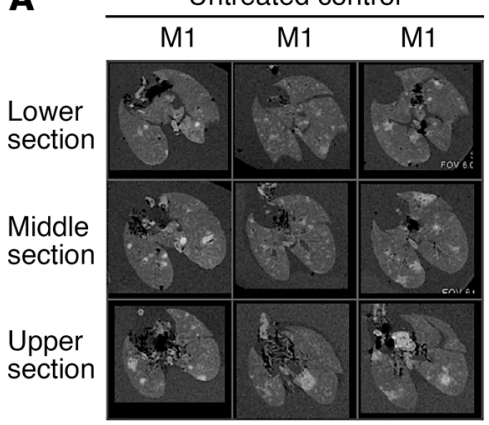

B

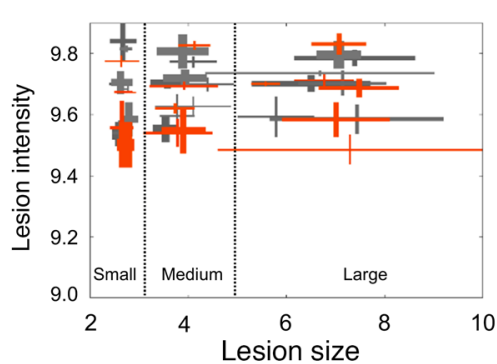

ISRIB treatment 4 weeks

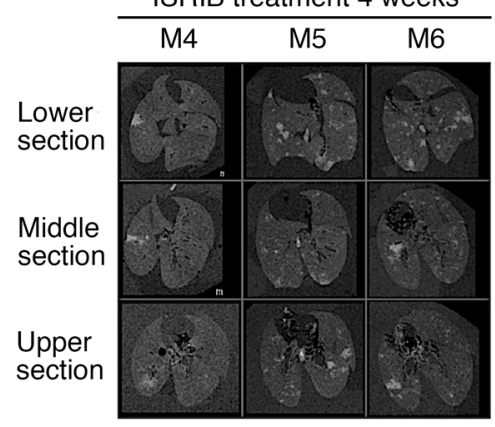

ISRIB 4 weeks

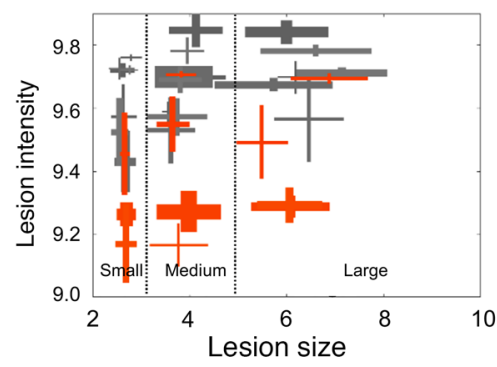

C
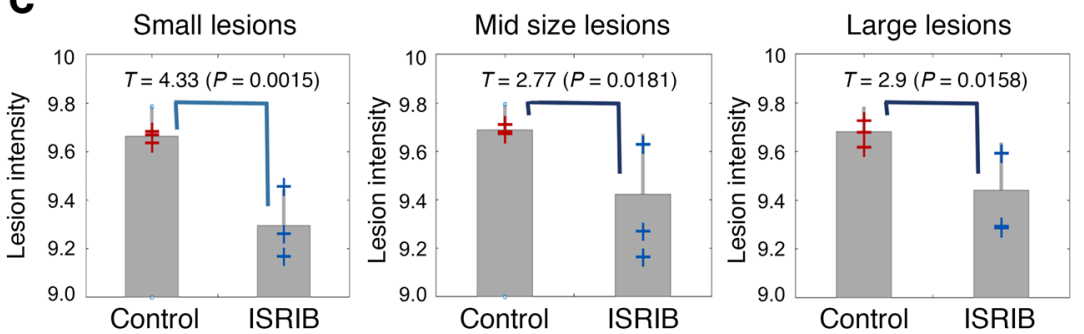

Figure 4. Global quantitative assessment of the ISRIB effect on pulmonary TB lesions using ex vivo MRI imaging. The B6.Sst $1^{5}$ mice were infected with $M$. tuberculosis by aerosol. The ISRIB was administered i.p. for 4 weeks starting at 4 weeks after infection. (A) Representative MRI sections of lungs at lower, middle, and upper levels of B6.Sst $1^{5}$ mice (M1-M6) at 8 weeks after infection ( 3 animals per group). Left panels = control mice; right panels = mice treated with ISRIB (1 mg/kg) for 4 weeks. (B) Distribution of size and intensity of individual lesions, as denoted by horizontal and vertical lines, respectively. Lung lesions of control (gray lines) and ISRIB-treated (red lines) B6.Sst $1^{5}$ mice treated for 24 (left panel) and 4 weeks (right panel) with ISRIB (1 mg/kg). (C) statistical analysis demonstrating the effect of the 4-week ISRIB treatment on the lesion intensity stratified by the lesion size.

most prominently by dsRNA. Traditionally, the PKR pathway has been associated with antiviral immunity, but more recently it was demonstrated that, in addition to viral dsRNAs, PKR can interact with and be activated by misfolded and dimerized endogenous RNA molecules $(32,33)$. Using the J2 antibody specific for dsRNA (34), we detected dsRNA speckles in the cytoplasm of TNF-stimulated BMDMs from both WT B6 and B6.Sst $1^{\mathrm{s}}$ mice (Supplemental Figure 1C). The presence of endogenous, cytosolic PKR ligands may provide an explanation for how IFN-induced PKR is activated by TNF even in noninfected macrophages. PKR activation by endogenous ligands has been linked to metabolic dysregulation via activation of the stress kinase JNK (35). We observed that JNK inhibition increased the expression of the ISR markers (Figure 2E), suggesting a role for the PKR/JNK-mediated feedback circuit proposed by Nakamura (33) in our model (see below).

These data demonstrate that in B6.Sst $1^{\mathrm{s}}$ macrophages TNF initiates a cascade of stress responses in a biphasic manner. At the early initiation phase (2-4 hours) we observed moderate ISR activation at similar levels in both the WT B6 and B6.Sst $1^{\mathrm{S}}$ mutant macrophages (Figure 2B). Corresponding to the early-phase ATF4 protein upregulation, XBP-1 splicing, known to be induced by IRE1 kinase activated specifically by ER stress, followed similar kinetics in both strains and peaked at 4-8 hours (Supplemental Figure 1D), suggesting that equally activated ER stress drives early ISR in WT B6 and B6.Sst1 ${ }^{\mathrm{s}}$ BMDMs. In support of this notion, the levels of the classical ER stress marker BiP were induced to similar levels in TNF-stimulated WT B6 and B6.Sst $1^{\mathrm{s}}$ macrophages in our microarray data set (NCBI GEO accession GSE99456). In the second phase, 12-24 hours following TNF stimulation, ISR activation increased exclusively in the B6.Sst $1^{\mathrm{s}}$ macrophages via a distinct IFN-I-dependent mechanism requiring PKR activation, whereas in contrast, ISR activation plateaued in WT B6 cells.

Proteomic changes induced by TNF reveal that Ipr1 upregulation in WT B6 macrophages correlates temporally with protection from escalating stress response. The major adaptive role of ISR is a global reduction of cap-dependent protein translation (27). However, translation of many proteins involved in stress responses proceeds via capindependent mechanisms, and the proportion of those proteins in the cellular proteome increases during prolonged stress. Thus, we postulated that an escalating ISR induced by TNF in B6.Sst $1^{\mathrm{s}}$ macrophages would result in global proteome remodeling. Therefore, we compared global quantitative protein abundance profiles of the WT $\mathrm{B} 6$ and $\mathrm{B} 6 . \mathrm{Sst} 1^{\mathrm{s}}$ mutant macrophages after stimulation with TNF, using stable isotope labeling of the digested macrophage proteomes with tandem mass tags followed by deep 2D LC-MS/ MS-based proteomic analysis.

The proteomic profiles of TNF-stimulated B6.Sst $1^{\mathrm{s}}$ and WT B6 macrophages were clearly distinct (Supplemental Table 3). A number of proteins were upregulated in the $\mathrm{B} 6 . \mathrm{Sst}^{\mathrm{s}}{ }^{\mathrm{s}}$ macrophages, demonstrating the absence of a total translational arrest in the mutant cells. In agreement with Hspa1a mRNA upregulation (Figure 1E), we detected higher levels of HSPA1A protein (see below). The TNF-stimulated B6.Sst $1^{\mathrm{s}}$ cells also expressed higher levels of ATF3, IFN-inducible proteins such as Rsad2, Cxcl10, Ifi35, Ifi47, Ifit1, Ifit2, Ifit3, and p47 GTPase, as well as proapoptotic proteins DAXX and Bim, cold shock-inducible RNA-binding protein $\mathrm{Rbm} 3$, and dsRNA-binding protein Stauphen. In contrast, the proteome of WT B6 macrophages stimulated with TNF was enriched in proteins involved in antioxidant defenses and protein homeostasis in the ER and cytoplasm, such as (a) NADHcytochrome b5 reductase 4 (CYB5R4), which protects cells from 
A

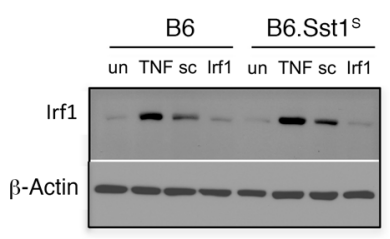

D

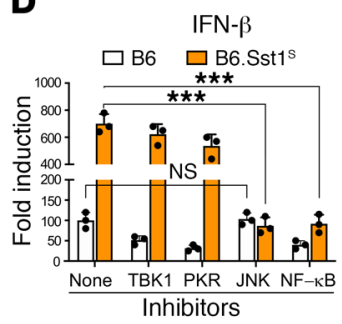

B

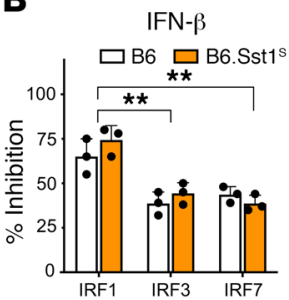

E

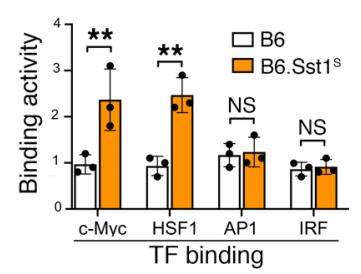

C

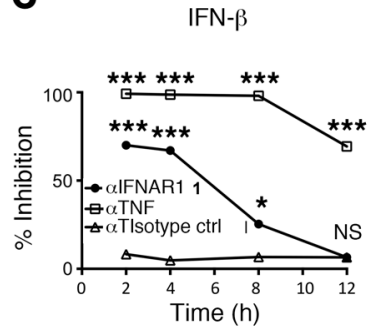

F

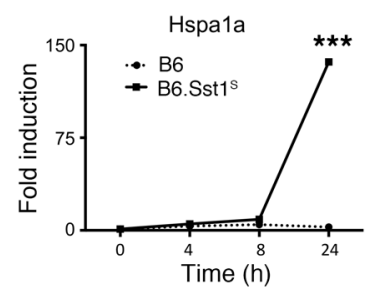

Figure 5. Transcriptional control of IFN- $\beta$ superinduction in B6.5st $1^{5}$ macrophages by TNF. (A) Effects of TNF stimulation and siRNA knockdown on IRF1 protein expression in WT $\mathrm{B} 6$ and B6.Sst ${ }^{\mathrm{S}} \mathrm{BMDMs}$ stimulated with $10 \mathrm{ng} /$ $\mathrm{mL}$ TNF for 24 hours. un, no TNF treatment; sc, scrambled siRNA control. (B) Inhibition of IFN- $\beta$ mRNA expression in TNF-stimulated WT B6

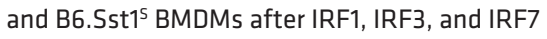
knockdown using siRNA. Percentage inhibition was calculated as compared to scrambled siRNA control. (C) Effects of TNF neutralization and IFNAR 1 blockade on IFN- $\beta$ mRNA expression in B6.Sst $1^{\text {s }}$ BMDMs treated with TNF for 16 hours. The anti-IFNAR1, anti-TNF- $\alpha$, and isotype control antibodies were added at time points indicated on the $x$ axis. (D) Effect of TBK1, PKR, JNK, and NF- $\kappa B$ inhibitors added after 12 hours of TNF stimulation on IFN- $\beta$ mRNA levels in $B 6.5 s t 1^{5}$ BMDMs treated at 16 hours. (E) Transcription factor (TF) binding activities in WT B6 and B6.Sst ${ }^{\mathrm{S}}$ BMDMs after TNF stimulation for 12 hours. (F

G

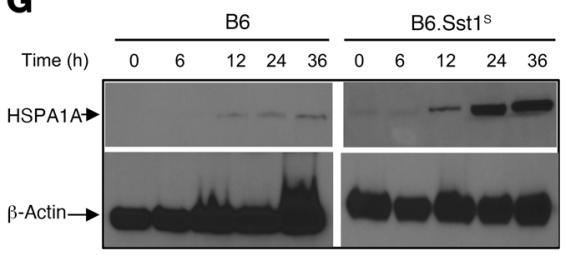

H

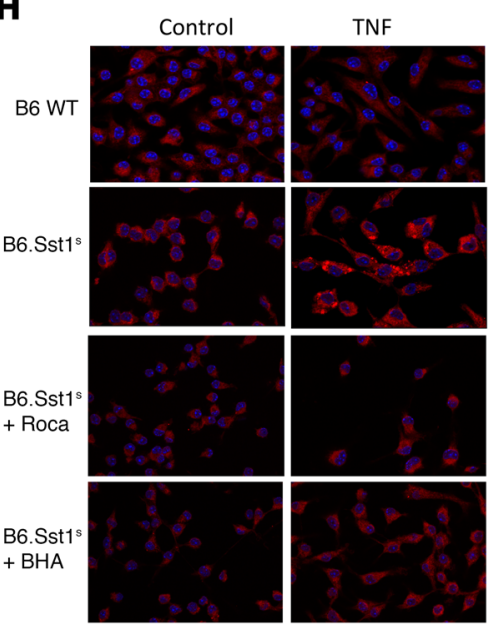

I

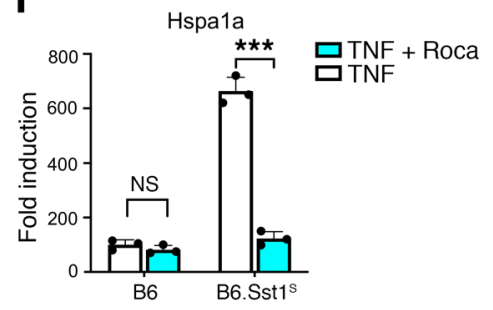

J
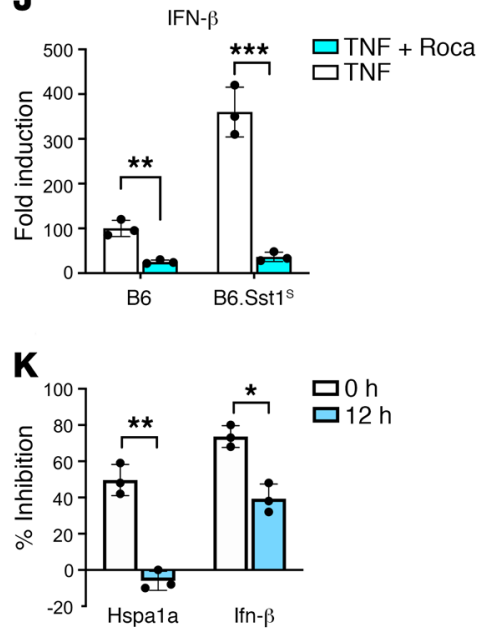

and $\mathbf{G ) ~ K i n e t i c s ~ o f ~ H s p a 1 a ~ m R N A ~ ( F ) ~ a n d ~ H S P A 1 A ~}$ protein (G) expression in WT B6 and B6.Sst ${ }^{5}$ BMDMs stimulated with TNF. (H) Aggresome formation in $\mathrm{B} 6$ and $\mathrm{B} 6.5 \mathrm{st} \mathrm{t}^{\mathrm{S}} \mathrm{BMDMs}$ stimulated with TNF for 24 hours. Lower panels = effects of the rocaglate $(50 \mathrm{nM})$ and $\mathrm{BHA}(100 \mu \mathrm{M})$ treatments on aggresome formation in B6.5st ${ }^{5}$ BMDMs. (I and J) Effects of rocaglate treatment (50 $\mathrm{nM}$ ) on superinduction of Hspa1a (I) and IFN- $\beta$ (J) mRNAs in TNF-stimulated B6.Sst $1^{\text {s }}$ BMDMs. (K) Suppression of the TNF-induced Hspa1a and IFN- $\beta$ mRNA upregulation in B6.Sst ${ }^{5}$ macrophages (at 18 hours) using BHA added at 0 or 12 hours of TNF treatment. Fold induction of gene expression in panels $\mathbf{D}, \mathbf{F}, \mathbf{I}$, and $\mathbf{J}$ was calculated relative to the mRNA expression in untreated B6 macrophages. In panels B-D, F, and I-K, 2-way ANOVA with Tukey's post hoc test was used on combined data of 3 independent experiments $\left({ }^{*} P=0.01-0.05 ;{ }^{* *} P<0.01 ;{ }^{* *} P<\right.$ $0.001)$. NS, not significant. excess buildup of ROS and oxidant stress (36); (b) stromal cellderived factor 2 (SDF2) involved in ER protein quality control, unfolded protein response, and cell survival under ER stress (37); (c) signal sequence receptor 2 (SSR2), a subunit of the ER TRAP complex involved in protein translocation across the ER membrane (38); and (d) stress-associated ER protein 1 (SERP1), which interacts with target proteins during their translocation into the lumen of the ER and protects unfolded target proteins against degradation during ER stress (39). Only the TNF-stimulated WT B6 cells upregulated the Sp110/Ipr1 and Sp140 proteins encoded within the sst 1 locus and implicated in TB susceptibility in vivo $(15,40)$. We extended these observations by finding that the Sp110/Ipr1 protein was induced in WT B6 macrophages between
8 and 12 hours after initial TNF stimulation, corresponding to a period of late stress escalation in the IPR1-negative B6.Sst1 ${ }^{\mathrm{s}}$ cells (Supplemental Figure 1E). Inhibition of JNK, p38, or IFNAR1 prevented IPR1 protein upregulation by TNF (Supplemental Figure 1F). Thus, Ipr1 is a stress- and IFN-inducible protein whose expression inversely correlated with the stress escalation and expression of IFN-I-inducible proteins, suggesting a role in preventing the IFN-mediated ISR in activated macrophages.

Small-molecule inhibition of the ISR reduces both susceptibility to M. tuberculosis and granuloma necrosis in vivo. Taken together, the above data demonstrate that compromised stress resilience of the B6.Sst ${ }^{\mathrm{s}}$ macrophages after TNF stimulation is mechanistically linked to unresolving ISR driven by IFN-I in a PKR- 


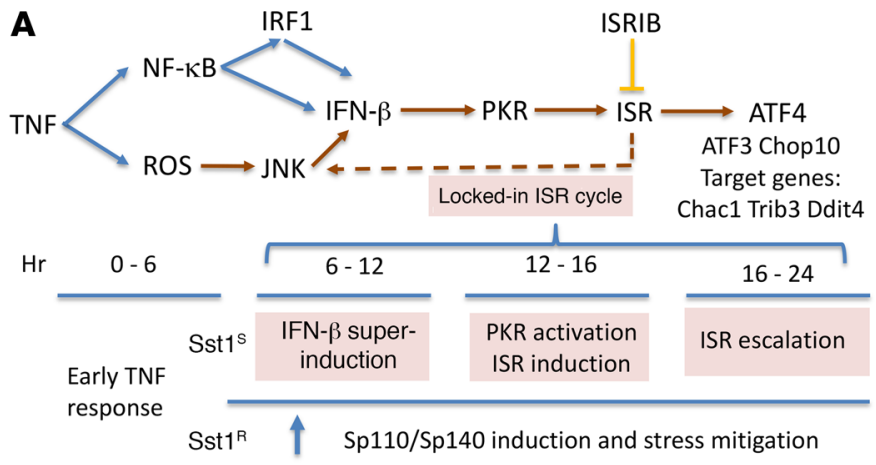

B

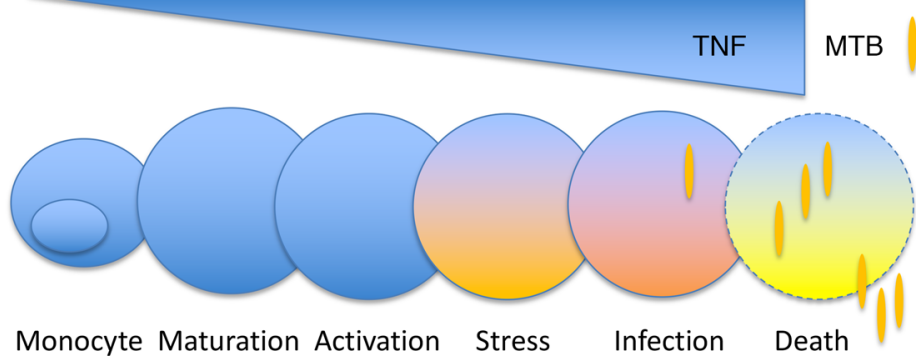

Figure 6. Mechanisms of TB susceptibility driven by TNF in $B 6.5 s 1^{5}$ mouse macrophages. (A) Aberrant activation of the integrated stress response (ISR) by TNF in $\mathrm{B} 6 . \mathrm{Sst}^{\mathrm{S}}{ }^{\mathrm{S}}$ macrophages via ROS- and proteotoxic stress-dependent superinduction of the type I IFN pathway. Blue lines = canonical TNF-activated pathways; red lines = mechanisms of IFN- $\beta$ superinduction by stress kinase JNK; red box $=$ PKR-mediated ISR activation and a hypothetical autoamplification loop. Sst1 ${ }^{R}$, sst1-resistant genotype. (B) Aberrant macrophage activation within inflammatory milieu of TB lesions prior to pathogen encounters promotes necrotic granuloma formation. MTB, Mycobacterium tuberculosis.

dependent manner. Therefore, we tested whether inhibitors of IFN-I and ISR pathways can correct the susceptible macrophage phenotype and, thus, decrease susceptibility to virulent $M$. tuberculosis in vivo.

First, we evaluated ISRIB, an inhibitor of eIF $2 \alpha$ phosphorylation that has been shown to reduce the ISR (41). Four weeks after aerosol infection with $M$. tuberculosis $\mathrm{H} 37 \mathrm{Rv}$, groups of mice were treated daily ( 5 out of 7 days per week) for 8 weeks with ISRIB at $0.25 \mathrm{mg} / \mathrm{kg}, 1.0 \mathrm{mg} / \mathrm{kg}$, or with vehicle control (Supplemental Figure 2A). The ISRIB treatment for 4 and 8 weeks significantly reduced lung bacterial loads (Figure $3 \mathrm{~A}$ ) and the degree of focal pneumonia with granuloma formation (Figure 3B and Supplemental Figure 2B). Two-dimensional evaluation of lung pathology with digitally scanned sections collected at both 4 and 8 weeks following the initiation of therapy revealed that both doses of ISRIB significantly reduced lung inflammation and necrosis (Figure $3 \mathrm{C}$ and Supplemental Figure 2C). To achieve a more global assessment of the impact of ISRIB therapy, we used 3D scanning with PET using the hypoxia-specific tracer $\left[{ }^{18} \mathrm{~F}\right] \mathrm{FMISO}$ alongside standard CT in live mice at 8 weeks of therapy. Quantitative, unbiased evaluation of non-vessel, non-airway, lung voxels associated with lung tissue consolidation revealed a trend toward reduced lung consolidation in the ISRIB-treated mice compared with untreated (Figure 3D).

We also assessed the extent of hypoxia within the cells of inflammatory foci since intracellular hypoxia is a known factor linking the ISR to the onset of necrosis (42). We used lesion-specific $\left[{ }^{18} \mathrm{~F}\right] \mathrm{FMISO}$ PET signals from our CT/PET scans since $\left[{ }^{18} \mathrm{~F}\right]$ FMISO is known to accumulate in the cytoplasm of hypoxic cells (43). Mice treated with the ISRIB showed a greater than 2-fold reduction in the mean internally normalized $\left.{ }^{18} \mathrm{~F}\right]$ FMISO lung lesion standardized uptake value signal compared with controls, supporting the conclusion that lesions from ISRIB-treated mice contained intracellular conditions that were less disposed to the onset of necrosis (Figure 3E).

In addition to the live imaging, we used magnetic resonance imaging (MRI) of the mouse lungs ex vivo (Figure 4). Mouse lungs were perfused with $4 \%$ paraformaldehyde in PBS and instilled with low-melt agarose in situ, as described in the Methods. We were able to detect lung lesions $200 \mu \mathrm{m}$ and greater in diameter. Representative sections are shown in Figure 4A. The MRI analyses using an in-house analytical pipeline was performed and the data for individual lesions were plotted, as shown in Figure 4B. This analysis parsed individual lesions according to their size and intensity. We observed that the ISRIB treatment for 4 weeks significantly reduced intensity of the lesions in each size category (Figure 4C). In agreement with the PET and histopathology data, this comprehensive structural analysis confirmed that ISRIB treatment reduced the inflammatory reaction within individual TB lesions irrespective of their size.

We also tested ISRIB for direct antibacterial activity against $M$. tuberculosis broth cultures and found no significant activity, with a minimal inhibitory concentration greater than $256 \mu \mathrm{g} / \mathrm{mL}$. On testing the ability of ISRIB to inhibit the proliferation of M. tuberculosis in B6 BMDMs, we found no antibacterial effect (Supplemental Figure 2D), confirming our hypothesis that ISRIB does not directly activate macrophage killing of $M$. tuberculosis. Instead, its actions in blunting the macrophage ISR pathway in vivo promote more balanced inflammatory and antimicrobial macrophage responses, resulting in reduced lung tissue damage.

Next, we tested the effect of ISRIB in a mouse model of more severe $\mathrm{TB}$ using $\mathrm{C} 3 \mathrm{HeB} / \mathrm{FeJ}$ mice that carry the $s s t 1^{s}$ allele on a genetic background that is more susceptible to M. tuberculosis (Supplemental Figure $3 \mathrm{~A})$. In this model, ISRIB $(0.25 \mathrm{mg} / \mathrm{kg})$ also significantly reduced lung $M$. tuberculosis burdens at 8 weeks after infection (Supplemental Figure 3B). In parallel, we assessed the mTOR pathway inhibition in this same mouse TB model, since this pathway has been reported to downregulate the mitochondrial ISR (44) and to induce autophagy (45). However, treatment with the mTor inhibitor sirolimus alone was ineffective (Supplemental Figure 3D). Because the $s s t 1^{S}$-mediated ISR is IFN-I driven, we tested the potent IFI-I pathway inhibitor amlexanox, which prevents phosphorylation of TBK1 and IKKe. Amlexanox treatment at both $25 \mathrm{mg} / \mathrm{kg}$ and 100 $\mathrm{mg} / \mathrm{kg}$ was inactive or even deleterious in terms of controlling lung M. tuberculosis loads and lung pathology (Supplemental Figure 3, $\mathrm{D}$ and E, respectively). This result suggests that the IFN-I-driven susceptibility mediated by the $s s t 1^{S}$ locus is independent of TBK1 and IKK $\varepsilon$ activation. Therefore, we wanted to delineate the mecha$\operatorname{nism}(\mathrm{s})$ of the IFN- $\beta$ upregulation in the $s s t 1^{S}$ background.

$P S$ drives the IFN- $\beta$ superinduction in a TBK1-independent manner. A previous report demonstrated that in WT B6 macrophages, 
TNF stimulated low levels of IFN- $\beta$ via NF- $\kappa \mathrm{B}$-mediated induction of IRF1, and that this was followed by autoamplification by secreted IFN- $\beta$ via IFNAR1 and IRF7 (26). In our model, the IRF1 protein was similarly upregulated by TNF in both WT B6 and B6.Sst1 ${ }^{\mathrm{s}}$ mutant macrophages (Figure 5A). To determine which of the IRF transcription factors might play a dominant role in the IFN-I pathway hyperactivation observed specifically in the B6.Sst $1^{\mathrm{S}}$ macrophages, we performed knockdowns of Irf1, Irf3, and Irf7 using siRNAs prior to stimulation of BMDMs with TNF (Figure 5A and Supplemental Figure 4, A-C). The Irf1 knockdown had the most pronounced effect, while the Irf3 and Irf7 knockdowns had similar but weaker effects on IFN- $\beta$ mRNA expression following 16 hours of TNF stimulation (Figure 5B). Importantly, knockdowns of any of these IRF1s reduced the IFN- $\beta$ expression proportionally in both WT and mutant macrophages and did not eliminate the strain differences in IFN- $\beta$ production (Supplemental Figure $4 \mathrm{C}$ ). Also, IFNAR1-blocking antibodies were ineffective in preventing the late-phase IFN- $\beta$ mRNA upregulation in the B6.Sst1 ${ }^{\mathrm{s}}$ cells when added after 8 hours of TNF stimulation, while TNF blockade remained efficient (Figure 5C), indicating that the late IFN- $\beta$ upregulation in the $s s t 1^{S}$ background required persistent TNF signaling and was not due to autoamplification by secreted IFN- $\beta$.

To identify pathway(s) specifically responsible for the latestage IFN- $\beta$ superinduction in the $B 6 . S s t 1^{\mathrm{s}}$ macrophages, we used small-molecule kinase inhibitors. We added these agents after 12 hours of TNF stimulation, and measured the IFN- $\beta$ mRNA levels 4 hours later (Figure 5D). Unsurprisingly, an NF- $\kappa$ B inhibitor (BAY11-7082) proportionally reduced the IFN- $\beta$ mRNA levels in both the WT and mutant macrophages. This observation supports the requirement for NF- $\mathrm{KB}$ and persistent TNF stimulation for the late-phase IFN- $\beta$ mRNA expression in both genetic backgrounds. Strikingly, inhibiting JNK completely eliminated the sst1-dependent difference; JNK inhibitor SP600125 reduced the IFN- $\beta$ mRNA expression in the B6.Sst $1^{\mathrm{S}}$ macrophages to the level of WT B6, but did not affect the IFN- $\beta$ expression level in WT B6 macrophages. In contrast, inhibition of another IFN-inducing kinase, TBK1, which is involved in signaling by nucleic acid recognition modules and IRF3 activation, affected the IFN- $\beta$ induction in both WT B6 and B6.Sst $1^{\mathrm{S}}$ cells to a much lesser degree and did not eliminate the strain differences in IFN- $\beta$ production (Figure 5D). These findings reveal that the sst1 locus exerts no effects on the canonical TNF/IRF1/IFN- $\beta$ axis and the IFN- $\beta$ / IFNAR1/IRF7/IFN- $\beta$ autoamplification loop, nor does it control the TBK1/IRF3 pathway. Rather, the late-phase superinduction of IFN- $\beta$, exclusively observed in B6.Sst $1^{\mathrm{s}}$ macrophages, results from synergy of the stress-activated kinase JNK with the canoni-

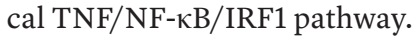

To gain deeper insight into sst1-mediated transcriptional regulation at this critical transition period, we compared transcription factor (TF) activities in WT $\mathrm{B} 6$ and $\mathrm{B} 6 . \mathrm{Sst}^{\mathrm{s}}$ macrophages following 12 hours of TNF stimulation using a TF activation array. The activities of NF- $\mathrm{B}, \mathrm{AP} 1$, STAT1, GAS/ISRE, IRF, NFAT, NFE2, CREB, YY1, and SP1 were upregulated by TNF to a similar degree in both the WT B6 and B6.Sst $1^{\text {s }}$ macrophages, while the HSF1 and MYC consensus sequence binding was significantly upregulated in the B6.Sst1 $1^{\mathrm{s}}$ mutant cells (Figure 5E). The increased activity of heat shock factor 1 (HSF1) in TNF- stimulated B6.Sst1 ${ }^{\mathrm{S}}$ macrophages is consistent with increased Hspa1a mRNA (Figure 1E) and protein levels (Supplemental Table 3), as compared with the WT B6 cells.

$P S$ induces protein aggregates in B6.Sst $1^{S}$ cells that can be rescued by the translation inhibitor rocaglate. The HSF1 TF activation and heat shock protein induction by TNF are indicative of PS. The kinetics of the Hspa1a mRNA expression demonstrated that in the WT cells the PS response was moderate. In contrast, it dramatically increased from 8 to 24 hours of TNF stimulation in the B6.Sst1 ${ }^{\mathrm{s}}$ cells (Figure 5F). The HSF1 inhibitor KRIB11, which blocks HSF1 activity, induced death of TNF-stimulated macrophages irrespective of their sst1 genotype (Supplemental Figure 5A), demonstrating that the HSF1-mediated stress response was an important survival pathway in TNF-stimulated macrophages of both backgrounds. However, progressive unresolving PS was observed only in B6.Sst $1^{\mathrm{S}}$ macrophages, as evidenced by robust upregulation and sustained expression of HSPA1A protein from 12 to 36 hours of TNF stimulation (Figure $5 \mathrm{G}$ ). We also documented accumulation of protein aggregates in the B6.Sst $1^{\mathrm{S}}$ cells during this period (Figure $5 \mathrm{H}$ ).

To further evaluate the role of PS escalation in the IFN- $\beta$ superinduction observed in B6.Sst1 ${ }^{\mathrm{S}}$ macrophages, we tested the impact of small-molecule derivatives of rocaglate that selectively inhibit cap-dependent protein translation by binding to the IF4A helicase subunit of a translation initiation complex $(46,47)$. Indeed, the rocaglate treatment inhibited protein biosynthesis in TNFstimulated macrophages without killing them (Supplemental Figure $5 \mathrm{~B}$ ). It also prevented the accumulation of protein aggregates (Figure 5H and Supplemental Figure 5C) and the Hspa1a mRNA upregulation in $\mathrm{B} 6 . \mathrm{Sst}^{\mathrm{S}}$ macrophages after TNF stimulation (Figure 5I). Remarkably, the rocaglate treatment also eliminated the difference between the TNF-stimulated WT B6 and B6.Sst1 ${ }^{\mathrm{S}}$ macrophages in IFN- $\beta$ mRNA expression (Figure 5J). Thus, preventing PS with rocaglate treatment also thwarted the IFN- $\beta$ superinduction. In contrast, the IFNAR1 blockade using neutralizing antibodies did not prevent the PS escalation (data not shown). We concluded that greater PS induced by TNF in B6.Sst1 ${ }^{\mathrm{S}}$ macrophages was upstream of the IFN- $\beta$ superinduction.

ROS are known to induce protein misfolding and PS. As shown in Supplemental Figure 5E, TNF stimulation induced ROS production by BMDMs in our model as well. Therefore, we tested whether ROS were involved in the PS induction. Indeed, we found that pretreatment of B6.Sst1 ${ }^{\mathrm{s}}$ macrophages with butylated hydroxyanisole (BHA) to boost their antioxidant defenses prior to TNF stimulation prevented protein aggregation (Figure $5 \mathrm{H}$ and Supplemental Figure 5C), inhibited superinduction of both Hspa1a and IFN- $\beta$ mRNAs (Figure 5K), and prevented subsequent ISR escalation (Supplemental Figure 5D). However, adding BHA at 12 hours of TNF stimulation had no inhibitory effect on the PS and only partially inhibited the IFN- $\beta$ mRNA expression (Figure $5 \mathrm{~K}$ ). The ISR escalation was also insensitive to the late treatment with BHA (Supplemental Figure 5D). These data demonstrate that TNFinduced ROS serve as a trigger of the aberrant TNF response in the B6.Sst $1^{\mathrm{s}}$ macrophages, causing protein misfolding and aggregation in the cytoplasm and subsequent escalation of the PS. The levels of ROS produced by TNF-stimulated WT B6 and B6.Sst1 ${ }^{\mathrm{s}}$ macrophages, however, were similar (Supplemental Figure 5E), 
suggesting that the B6.Sst1 ${ }^{\mathrm{S}}$ cells differ from the WT B6 macrophages in their adaptation to ROS-mediated stress.

\section{Discussion}

Our research reveals that the sst1-mediated susceptibility to $M$. tuberculosis and the concomitant development of the necrotic granuloma is mechanistically linked to an aberrant macrophage response to TNF and sustained escalating stress responses. Because the $s s t 1^{S}$ phenotype in mice closely resembles the pathology of human $\mathrm{TB}$, this study provides a rationale for testing whether the aberrant macrophage response to TNF is also associated with progression of TB in humans. In addition, our findings suggest a novel disease-modifying therapeutic strategy for correcting the aberrant TNF response, rather than blocking this essential mediator of host resistance.

TNF has been proven to be an essential cytokine for the formation and maintenance of TB granulomas and TB resistance both in humans and in animal models. However, it plays a dual role in host $-M$. tuberculosis interactions; excessive responses to TNF drive immunopathology in animal models and are associated with TB susceptibility in humans $(6,7)$. Our data demonstrate that an aberrant response of macrophages to TNF may drive necrotization of TB granulomas via a potentially novel IFN-I-mediated cascade. TNF-exposed susceptible macrophages undergo superinduction of the IFN-I pathway - at first initiated by oxidative stress and dysregulated proteostasis, and then subsequently driven by a JNK/IFN- $\beta /$ PKR circuit - that culminates with induction of the eIF $2 \alpha$-triggered ISR, an event that leads to a locked-in state of escalating ISR and, ultimately, macrophage death and tissue necrosis (Figure 6A). This mechanism provides a plausible explanation for the association of TB progression with hyperactivation of IFN-I and ISR observed in human TB patients $(8,25)$.

Our studies revealed that at an early stage, TNF stimulation caused modest triggering of the ISR in both WT and the $s s t 1^{s}$ mutant macrophages. At this early stage the ISR is, most likely, driven by the ER stress and unfolded protein response, as previously described for macrophage activation (48). The accumulation of unfolded proteins in the cytoplasm and ER induced by TNF leads to transient activation of the ISR via HRI and PERK kinases, respectively, to temporarily inhibit cap-dependent protein translation in order to reduce the protein overload and support macrophage survival (24). The unique, second wave of ISR activation is exclusively observed in B6.Sst $1^{\mathrm{s}}$ macrophages. This escalating stress proceeds via superinduction of IFN- $\beta$, activation of the IFN- $\beta /$ PKR/ISR axis, and subsequent upregulation of proapoptotic genes and proteins. This mechanism is similar to a signaling cascade recently described in a model of Listeria monocytogenes infection (49). However, in the B6.Sst1 ${ }^{\mathrm{s}}$ macrophages, the IFN- $\beta$ superinduction and ISR escalation did not require infection and was triggered by TNF alone. We excluded a significant contribution of the STING/TBK1/IRF3 pathway to the observed IFN- $\beta$ superinduction (Figure 5). These findings are consistent with a lack of effects of STING and IRF3 gene knockouts on mouse TB susceptibility in vivo $(23,50)$. Thus, the hyperactivation of the IFN-I pathway could be explained solely by a cooperative effect of persistent activation of the NF- $\kappa \mathrm{B}$ and JNK pathways. Both pathways are known to converge on IFN- $\beta$ enhancer elements and to recruit coactivators and chromatin remodeling proteins to form an enhanceosome (51).

JNK is a stress kinase that is activated in response to oxidative, proteotoxic, metabolic, and other challenges and is an important part of the cellular defense strategy (52). Whereas transient JNK activation is adaptive, prolonged JNK activation is known to contribute to a proapoptotic transition. Our studies suggest that this transition may be driven by IFN-mediated PKR activation and ISR escalation. Conversely, PKR itself has been shown to directly stimulate JNK activity in macrophages (35). This may occur by translational arrest via a mechanism known as the ribotoxic stress response (53). Of note, our recent studies have shown that inhibition of JNK and PKR in TNF-stimulated B6.Sst1 ${ }^{\text {s }}$ BMDMs using small molecules eventually results in further ISR escalation, which is driven by ROS and is due to defective antioxidant defense activation (E. Brownhill, personal communication). This response may be driven by a cytoplasmic unfolded protein response via another eIF2 $\alpha$ kinase, HRI (24). Therefore, we propose that the JNK/IFN- $\beta /$ PKR circuit is initially activated by oxidative stress as an adaptive mechanism to reduce protein translation and to decrease misfolded protein loads in the ER and cytoplasm. However, sustained upregulation of JNK, IFN- $\beta$, and PKR forms a maladaptive feed-forward stress response circuit, locking TNFstimulated B6.Sst1 $1^{\mathrm{s}}$ macrophages in a state of unresolving stress, as illustrated in Figure 6A.

In TNF-stimulated B6.Sst1 ${ }^{\mathrm{s}}$ macrophages IFN-I also drives the upregulation of the IFN-stimulated genes Rsad2 and Ch25h, whose products are known to inhibit mitochondrial function and lipogenesis, respectively $(49,54)$. In addition, the 25 -hydroxycholesterol produced by Ch25h enzymatic activity can further increase the ISR (55). Moreover, by limiting cholesterol biosynthesis it can sustain elevated IFN-I signaling (56) and amplify inflammatory cytokine production (57). We propose that coincidence of hyperinflammation with downregulation of essential metabolic pathways, both driven by IFN-I-mediated pathways, results in accumulation of damage in macrophages exposed to these cytokines in TB granulomas prior to infection and decrease their resilience to subsequent infection with intracellular bacteria (Figure 6B).

At the systemic level early in infection, IFN-I initially primes protective inflammatory responses, but has been shown to cause immunosuppression at later stages via induction of soluble mediators (12). Recently, IFN-I was shown to induce expression of IL-1Ra and, thus, suppress effector mechanisms of IL-1-mediated host resistance to $M$. tuberculosis (23). Our studies demonstrate cellautonomous effects of IFN-I in macrophages of susceptible hosts, where IFN-I exacerbates inflammatory damage via PKR-mediated ISR and downregulation of metabolic pathways essential for macrophage repair and stress resilience. Of note, recent analysis of human monocyte-derived macrophages after infection with $M$. tuberculosis in vitro using single-cell gene expression profiling revealed that an ISR marker, ATF3, and IL-1Ra were expressed by the same cells (58). These observations, alongside our current studies of sst1-mediated mechanisms of susceptibility to TB in the B6.Sst $1^{\text {s }}$ mouse, provide an explanation of a dual role of IFN-I in host interactions with $M$. tuberculosis $(9,59)$.

An important observation in this study is that ISR inhibition with the small molecule ISRIB showed a therapeutic benefit 
against $M$. tuberculosis proliferation and granuloma formation in infected B6.Sst1 ${ }^{\mathrm{s}}$ mice. Interestingly, we found that ISRIB dosed daily at $0.25 \mathrm{mg} / \mathrm{kg}$ demonstrated superior inhibition of M. tuberculosis proliferation in $\mathrm{B} 6 . \mathrm{Sst}^{\mathrm{s}}$ mice than did $1.0 \mathrm{mg} / \mathrm{kg}$. Moreover, even higher doses $(5 \mathrm{mg} / \mathrm{kg}$ ) were deleterious (Supplemental Figure 3B). Parenterally administered ISRIB has been shown to inhibit eIF2 $\alpha$ phosphorylation with an $\mathrm{IC}_{50}$ of $5 \mathrm{nM}$, and when given to mice it achieves its $\mathrm{C}_{\max }$ at 2 hours and has a $t_{1 / 2}$ of 8 hours (60). In cell-based studies it has shown typical direct doseresponse relationships (60-62). Animal studies characterizing its efficacy for memory or traumatic brain injury have typically used doses of $2.5 \mathrm{mg} / \mathrm{kg}$, and these evaluations have also shown direct dose-response relationships $(60,63)$. Although further research will be necessary to explain the inverse dose-response relationship we observed, it is worth noting that previous animal studies with ISRIB administered the drug for durations of up to 28 days, while our study gave the drug for 8 weeks in the presence of a chronic infection. Hence it is conceivable that high-dose ISRIB may have deleterious off-target effects that manifest with long-term dosing or the presence of chronic inflammation. These data warrant further investigation of the effects of dosage, timing, and duration of ISRIB administration on TB granuloma dynamics.

The sst 1 locus encodes the IFN-inducible nuclear protein Sp110. In WT macrophages, this protein is induced during the late stage of TNF response between 8 and 12 hours; however, Sp110 expression is absent in TNF- or IFN- $\gamma$-activated macrophages carrying the $s s t 1^{s}$ allele (15). This late-stage time interval — which precedes the escalation of the PS and superinduction of IFN- $\beta-$ is when we first observe manifestations of the susceptible phenotype, and during this period, Sp110 is known to accumulate in the nucleus and to associate with chromatin in mouse and human macrophages (B. Bhattacharya, unpublished observations). These observations suggest that Sp110 plays a key role in governing macrophage stress resilience probably by controlling chromatin organization and function.

Recently, the function of Sp140 (another Sp100 family member also encoded within the sst1 locus) has been elucidated (19). In activated macrophages, Sp140 - an IFN-inducible chromatinbinding bromodomain protein - plays an important role in preserving a macrophage-specific transcriptional program by binding to promoters of lineage-inappropriate repressed genes and maintaining their repressed status. Among those was the developmental HOXA9 gene whose activity preserves hematopoietic stem cell self-renewal and suppresses macrophage differentiation. Knockdown of Sp140 leads to derepression of the HOXA9 gene and aberrant macrophage activation, including an upregulation of Myc- and E2F-regulated gene sets. In vivo, Sp140 downregulation results in exacerbated inflammatory colitis. In humans, Sp140 polymorphisms have been associated with Crohn's disease and multiple sclerosis in GWAS (reviewed in ref. 21). The Sp110 polymorphisms have been associated with severity of canine degenerative myelopathy, a neurodegenerative disease with similarities to human ALS (20). We hypothesize that in activated macrophages the Sp100 family members play a central role in crosstalk between stress- and IFN-mediated pathways to maintain macrophage differentiation and activation programs and boost macrophage stress resilience. This paradigm would explain the broad roles of those proteins in limiting inflammation-associated tissue damage initiated by infectious and noninfectious triggers.

The studies of the sst1-mediated susceptibility in mice revealed a mechanistic link between an IFN-I-dominated hyperinflammatory response and local necrotic immunopathology in TB granulomas. This mouse model permits further analysis of local lung- and granuloma-specific mechanisms that enable TB progression in immune-competent hosts. Our studies demonstrate that macrophage susceptibility emerges gradually within inflammatory tissue due to an imbalance of macrophage stress responses. Unresolving stress induced by TNF and exacerbated by IFN-I may lead to functional decline and suppression of bactericidal immune responses in macrophages prior to contact with microbes. By exploiting this regulatory failure, successful pathogens, such as M. tuberculosis, may bypass mechanisms of resistance locally in otherwise immune-competent hosts. This strategy would ensure survival of both the host and the pathogen and facilitate successful transmission of the later.

The escalating stress response mechanism leading to necrosis during $\mathrm{TB}$ represents an attractive therapeutic target. Indeed, this study reveals that a small-molecule inhibitor of the ISR, ISRIB, is effective in preventing necrosis in M. tuberculosis-infected mouse lungs and concomitantly restricts $M$. tuberculosis proliferation. ISRIB has demonstrated promising results in enhancing memory, preventing neuronal degeneration, and reducing tumor growth $(64,65)$. Further studies in combination with traditional anti-TB drugs may define whether future ISR inhibitors that are being developed for human use may offer therapeutic benefit as a specific necrotic granuloma-directed therapy for TB.

\section{Methods}

Reagents. Recombinant mouse TNF was from Peprotech and recombinant mouse IL-3 was from R\&D Systems. Mouse monoclonal antibody against mouse TNF (clone XT22) was from Thermo Fisher Scientific and isotype control and mouse monoclonal antibody against mouse IFN- $\beta$ (clone MAR1-5A3) was from eBioscience. ATF4 (sc-39063), ATF3 (sc-518032), mouse monoclonal anti-PKR (sc-6282), and rabbit polyclonal anti-p-PKR (sc-101783) were obtained from Santa Cruz Biotechnology. Anti-HSPA1A (A-400) and- $\beta$-actin (A1978) antibodies were obtained from R\&D Systems and MilliporeSigma, respectively. Anti-IRF1 and -IRF3 antibodies were obtained from Cell Signaling Technology. The Ipr1-specific rabbit antiserum was generated by Covance Research Products, Inc. (66). The Ipr1 monoclonal antibodies were generated using Ipr1 peptides from Abmart and validated in our laboratory. Inhibitors BAY 11-7082, phenylbutyrate sodium (PBA), and rapamycin were from Enzo Life sciences. SB203580, SP600125, and C16 were obtained from Calbiochem. JQ1, flavopiridol, and 10058-F4 were from Tocris. ISRIB, poly(I:C), LPS from E. coli (055:B5), triptolide, and BHA were obtained from MilliporeSigma. BX-795 was from Invivogen. RHT was provided by Aaron Beeler (Boston University).

Animals. C57BL/6J and $\mathrm{C} 3 \mathrm{HeB} / \mathrm{Fe}$ inbred mice were obtained from the Jackson Laboratory. The C3H.B6-sst1, C3H.scid, and C3H.B6-sst1 SCID mouse strains were generated in our laboratory as described previously (15, 17, 22). The B6.C3H-sst1(B6J.C3-sst1 ${ }^{\text {C3HeB/Fej Krmn) }}$ congenic mice were created by transferring the $s s t 1^{S}$ allele from $\mathrm{C} 3 \mathrm{HeB} /$ FeJ mouse strain on the $\mathrm{B} 6(\mathrm{C} 57 \mathrm{BL} / 6 \mathrm{~J})$ genetic background using 12 backcrosses (referred to here as B6.Sst1 ${ }^{\mathrm{S}}$ ). 
$B M D M$ culture. Isolation of mouse bone marrow and culture of BMDMs were carried out as previously described (66). TNF-activated macrophages were obtained by culture of cells for various times with recombinant mouse TNF (10 ng/mL). Cells were washed again and cultured in the presence of inhibitors and TNF in DMEM/F12 containing $10 \% \mathrm{FBS}$ without antibiotics at $37^{\circ} \mathrm{C}$ in $5 \% \mathrm{CO}_{2}$ for 24 hours.

Animal infections and imaging. Mice were infected by $M$. tuberculosis H37Rv using a Glas-Col chamber, and mice were sacrificed for enumeration of $M$. tuberculosis $\mathrm{CFU}$ in lungs and spleen day 1 counts as well as subsequent time points as previously described (67). H\&Estained tissue sections were imaged by high-resolution digital microscopy and lesion scoring was performed as described previously (68). High-resolution PET/CT imaging was conducted using a Mediso nanoScan instrument. Image analysis to quantify disease burden in lungs was performed using previously reported algorithms (69).

Ex vivo MRI. MRI was performed on fixed lungs using a $4.7-\mathrm{T}$ Bruker MRI scanner. Lungs were perfused with $4 \%$ paraformaldehyde in PBS and inflated with 1\% low-melt agarose in PBS. A high-resolution 3D structural MRI (FLASH) sequence was applied for imaging the lungs to acquire micron-scale images (down to $0.1 \mathrm{~mm}$ voxel size). Lesion classification was performed using an in-house lesion segmentation pipeline based on the expectation-maximization (EM) algorithm (70), which is widely used in the neuroimaging field. The pipeline automatically separates individual lesions based on spatial voxel connectivity information and reports total number of lesions, mean intensity profile of each lesion, and 3D volumetry (size in 3D) of each lesion per subject. Statistical analysis: the ISRIB treatment and control groups were compared on the basis of total number and intensity of lesions in small, medium, and large size categories using a nonparametric Kolmogorov-Smirnov or Friedman test.

Immunoblotting. To monitor Ipr1 protein levels we developed Ipr1 peptide-specific rabbit polyclonal antibodies, which recognize the Ipr1 protein of predicted length on Western blots (71). BMDMs were subjected to treatments specified in the text. Nuclear extracts were prepared using the nuclear extraction kit from Signosis. Whole-cell extracts were prepared by lysing the cells in RIPA buffer supplemented with protease inhibitor cocktail and phosphatase inhibitor I and III (MilliporeSigma). Equal amounts $(30 \mu \mathrm{g})$ of protein from whole-cell extracts were resolved by SDS-PAGE and transferred to PVDF membranes (Millipore). After blocking with 5\% skim milk in TBS-T (20 mM Tris- $\mathrm{HCl}$ [pH 7.5], $150 \mathrm{mM} \mathrm{NaCl}$, and $0.1 \%$ Tween 20) for 2 hours, the membranes were incubated with the primary antibody overnight at $4^{\circ} \mathrm{C}$. Protein bands were detected with an enhanced chemiluminescence (ECL) kit (PerkinElmer). Stripping was performed using Western blot stripping solution (Thermo Fisher Scientific). The loading control $\beta$-actin (MilliporeSigma, 1:2000) was evaluated on the same membrane. The Ipr1-specific rabbit antiserum was generated by Covance Research Products, Inc. (1:500) as described previously (66). The Ipr1 monoclonal antibodies were generated using Ipr1 peptides from Abmart. ATF4, ATF3, Gadd34, c-Myc, Daxx, p21, PKR, and phospho-PKR antibodies were obtained from Santa Cruz Biotechnology; rabbit polyclonal anti-pPKR (sc101783, Santa Cruz Biotechnology) and mouse monoclonal anti-PKR (sc6282, Santa Cruz Biotechnology) were used at dilution factor of 1:150 and 1:200, respectively.

IRF1, IRF3 (1:1000), p38, p-p38, JNK, and p-JNK antibodies were obtained from Cell Signaling Technology. Anti-HSPA1A (1:1000) antibody was obtained from R\&D Systems. Anti- $\beta$-actin antibody (1:2000) was obtained from MilliporeSigma.

RNA isolation and $q P C R$. Total RNA was isolated using the RNeasy Plus Mini Kit (Qiagen). cDNA synthesis was performed using SuperScript II (Invitrogen). Real-time PCR was performed with GoTaq qPCR Master Mix (Promega) using the CFX-90 real-time PCR System (Bio-Rad).Oligonucleotide primers were designed using Primer 3 software (https://bioinfo.ut.ee/primer3-0.4.0/) (Supplemental Table 1) and specificity was confirmed by melting curve analysis. Thermal cycling parameters involved 40 cycles under the following conditions: $95^{\circ} \mathrm{C}$ for 2 minutes, $95^{\circ} \mathrm{C}$ for 15 seconds, and $60^{\circ} \mathrm{C}$ for 30 seconds. Each sample was set up in triplicate and normalized to RPS17 or $18 \mathrm{~S}$ expression by the $\Delta \Delta \mathrm{Ct}$ method.

Immunofluorescence microscopy. Cells were fixed with $4 \%$ paraformaldehyde for 15 minutes at room temperature, permeabilized with $0.25 \%$ Triton X-100 for 30 minutes, and then blocked for 20 minutes with $2.5 \%$ goat serum. Cells were incubated with primary antibodies (mouse monoclonal antibodies against J2 [1:3000] overnight at $4^{\circ} \mathrm{C}$ in $2.5 \%$ goat serum), and then incubated with Alexa Fluor 488-conjugated (excitation/emission maxima 490/525 nm) donkey anti-mouse IgG (1:1000, Invitrogen) secondary antibody for 2 hours. Images were acquired using a Leica SP5 confocal microscope. All images were processed using Image J software (NIH).

Hoechst/PI staining method for cell cytotoxicity. For cell viability assays, BMDMs were plated in 96-well tissue culture plates $(12,000$ cells/well) in phenol red-free DMEM/F12 media and subjected to necessary treatments. Hoechst (Invitrogen, $10 \mu \mathrm{M}$ ) and PI (Calbiochem, $2 \mu \mathrm{M}$ ) were added and the cells incubated at $37^{\circ} \mathrm{C}$ for 15 minutes and read in a Celigo Imaging Cytometer. The percentage of total and dead cells was calculated for each treatment.

TF profiling analysis. Each array assay was performed following the procedure described in the $\mathrm{TF}$ activation profiling plate array kit user manual (Signosis, FA-001). Ten micrograms of nuclear extract was first incubated with the biotin-labeled probe mix at room temperature for 30 minutes. The activated TFs were bound to the corresponding DNA-binding probes. After the protein-DNA complexes were isolated from unbound probes, the bound probes were eluted and hybridized with the plate precoated with the capture oligonucleotides. The captured biotin-labeled probes were then detected with streptavidin-HRP and subsequently measured with a TECAN microplate reader.

siRNA knockdown. Gene knockdown was done using GenMute (SignaGen) and FlexiTube GeneSolution siRNAs from Qiagen. AllStars negative control siRNA (SI03650318) from Qiagen was used as a negative control. BMDMs (B6.Sst $1^{\mathrm{s}}$ and WT) were seeded into 6-well plates at a density of $2.5 \times 10^{5}$ per well and grown as described above. Shortly before transfection, the culture medium was removed and replaced with $1 \mathrm{~mL}$ complete medium, and the cells were returned to normal growth conditions. To create transfection complexes, 15 nM siRNA (pool of 4 siRNAs) in 1× GenMute buffer (total $500 \mathrm{~mL}$ ) was incubated with $1.5 \mu \mathrm{L}$ of GeneMute transfection reagent for 15-20 minutes at room temperature. The complexes were added drop-wise onto the cells. Cells were incubated with the transfection complexes for 24 hours at $37^{\circ} \mathrm{C}$ in $5 \% \mathrm{CO}_{2}$. After 24 hours, cells were washed to remove siRNA and replenished with fresh media. TNF (10 ng/mL) was added for 24 hours and BMDMs were harvested as outlined below. siRNA pools included Irf1 (GS16362), Irf3 (GS54131), and Irf7(GS54123). 
ELISA. Supernatants were collected from mouse macrophages after 24 hours of stimulation with TNF- $\alpha$ or poly(I:C). IFN- $\beta$ was measured using the mouse IFN- $\beta$ ELISA kit from PBL Assay Science. ELISAs were done as recommended by the manufacturer.

Statistics. The data were analyzed using a nonparametric 2-tailed Mann-Whitney test, Welch's $t$ test, or ANOVA with post hoc tests for multiple comparisons as denoted in figure legends. In animal experiments each data point represents a survey of 1 mouse, and all $P$ values were calculated based on a nonparametric ANOVA (Kruskal-Wallis test) comparing the control to each of the dosing groups while accounting for multiple comparisons. All tests were run using GraphPad Prism 8. $P$ values less than 0.05 were considered significant.

Study approval. All experiments were performed with the full knowledge and approval of the Standing Institutional Animal Care and Use Committee (IACUC) and Institutional Biosafety Committee (IBC) at Boston University (IACUC protocol PROTO201800218, IBC protocol 19-875) and Johns Hopkins University (IACUC protocol MO19M98, IBC protocol P9902170221) in accordance with relevant guidelines and regulations.

\section{Author contributions}

$\mathrm{BB}, \mathrm{ARI}, \mathrm{SJ}, \mathrm{LK}, \mathrm{WB}$, and IK designed research studies. BB, SX, SC, MU, AO, EAI, GA, SL, RB, AP, YG, BBK, AP, and BSY con- ducted experiments and performed data acquisition. BB, SX, MU, AO, EAI, GA, RB, LK, BBK, SJ, WB, and IK analyzed the data and prepared figures and publication materials. JC provided reagents and advice for PKR analysis. BB, MU, LK, WB, and IK wrote the manuscript.

\section{Acknowledgments}

This work was sponsored by NIH grants R01 HL133190 and R01 HL126066 (to IK and WRB), and by the National Center for Advancing Translational Sciences, NIH, through BU-CTSI grant number 1UL1TR001430. The authors are grateful to Robert Silverman, Karen Elkins, and Benjamin Wolozin for helpful discussions; to Adam Gover and Somak Ray for the analyses of microarray and proteomics data, respectively; to Kathleen Mulka for assistance with histologic scoring and quantification; and to Shivraj Yabaji for help with data analysis and manuscript preparation.

Address correspondence to: William R. Bishai, MD, PhD, Johns Hopkins School of Medicine, 1550 Orleans St., Baltimore, Maryland 21287, USA. Phone: 510.955.3507; Email: wbishai@jhmi. edu. Or to: Igor Kramnik, MD, PhD, Boston University School of Medicine, NEIDL, 620 Albany St., Boston, Massachusetts 02118, USA. Phone: 617.358.9187; Email: ikramnik@bu.edu.
1. Kramnik I, Beamer G. Mouse models of human TB pathology: roles in the analysis of necrosis and the development of host-directed therapies. Semin Immunopathol. 2016;38(2):221-237.

2. Cadena AM, et al. Heterogeneity in tuberculosis. Nat Rev Immunol. 2017;17(11):691-702.

3. Pajuelo D, et al. NAD hydrolysis by the tuberculosis necrotizing toxin induces lethal oxidative stress in macrophages. Cell Microbiol. 2019;22(1):e13115.

4. Amaral EP, et al. A major role for ferroptosis in Mycobacterium tuberculosis-induced cell death and tissue necrosis. J Exp Med. 2019;216(3):556-570.

5. Wallis RS, Ehlers S. Tumor necrosis factor and granuloma biology: explaining the differential infection risk of etanercept and infliximab. Semin Arthritis Rheum. 2005;34(5):34-38.

6. Tobin DM, et al. Host genotype-specific therapies can optimize the inflammatory response to mycobacterial infections. Cell. 2012;148(3):434-446.

7. Roca FJ, Ramakrishnan L. TNF dually mediates resistance and susceptibility to mycobacteria via mitochondrial reactive oxygen species. Cell. 2013;153(3):521-534.

8. Berry MPR, et al. An interferon-inducible neutrophil-driven blood transcriptional signature in human tuberculosis. Nature. 2010;466(7309):973-977.

9. Moreira-Teixeira L, et al. Type I interferons in tuberculosis: Foe and occasionally friend. J Exp Med. 2018;215(5):1273-1285.

10. Scriba TJ, et al. Sequential inflammatory processes define human progression from $M$. tuberculosis infection to tuberculosis disease. PLoS Pathog. 2017;13(11):e1006687.

11. Zak DE, et al. A blood RNA signature for tuberculosis disease risk: a prospective cohort study.
Lancet. 2016;387(10035):2312-2322.

12. McNab F, et al. Type I interferons in infectious disease. Nat Rev Immunol. 2015;15(2):87-103.

13. Kramnik I, et al. Genetic control of resistance to experimental infection with virulent Mycobacterium tuberculosis. Proc Natl Acad Sci US A. 2000;97(15):8560-8565.

14. Lanoix J-P, et al. Heterogeneous disease progression and treatment response in a $\mathrm{C} 3 \mathrm{HeB} / \mathrm{FeJ}$ mouse model of tuberculosis. Dis Model Mech. 2015;8(6):603-610.

15. Pan $\mathrm{H}$, et al. Ipr1 gene mediates innate immunity to tuberculosis. Nature. 2005;434(7034):767-772.

16. Sissons J, et al. Multigenic control of tuberculosis resistance: analysis of a QTL on mouse chromosome 7 and its synergism with sst1. Genes Immun. 2009;10(1):37-46.

17. Pichugin AV, et al. Dominant role of the sst1 locus in pathogenesis of necrotizing lung granulomas during chronic tuberculosis infection and reactivation in genetically resistant hosts. Am J Pathol. 2009;174(6):2190-2201.

18. Leu JS, et al. SP110b controls host immunity and susceptibility to tuberculosis. Am J Respir Crit Care Med. 2017;195(3):369-382.

19. Mehta S, et al. Maintenance of macrophage transcriptional programs and intestinal homeostasis by epigenetic reader SP140. Sci Immunol. 2017;2(9):eaag3160.

20. Ivansson EL, et al. Variants within the SP110 nuclear body protein modify risk of canine degenerative myelopathy. Proc Natl Acad Sci U S A. 2016;113(22):E3091-E3100.

21. Fraschilla I, Jeffrey KL. The speckled protein (SP) family: immunity's chromatin readers. Trends Immunol. 2020;41(7):572-585.

22. Yan BS, et al. Progression of pulmonary tuberculosis and efficiency of bacillus Calmette-Guérin vaccination are genetically controlled via a common sst1-mediated mechanism of innate immunity. JImmunol. 2007;179(10):6919-6932.

23. Ji DX, et al. Type I interferon-driven susceptibility to Mycobacterium tuberculosis is mediated by IL-1Ra. Nat Microbiol. 2019; 4(12):2128-2135.

24. Abdel-Nour M, et al. The heme-regulated inhibitor is a cytosolic sensor of protein misfolding that controls innate immune signaling. Science. 2019;365(6448):eaaw4144.

25. Seimon TA, et al. Induction of ER stress in macrophages of tuberculosis granulomas. PLoS One. 2010;5(9):e12772.

26. Yarilina A, et al. TNF activates an IRF1dependent autocrine loop leading to sustained expression of chemokines and STAT1-dependent type I interferon-response genes. Nat Immunol. 2008;9(4):378-387.

27. Lu PD, et al. Cytoprotection by pre-emptive conditional phosphorylation of translation initiation factor 2. EMBO J. 2004;23(1):169-179.

28. Taniuchi S, et al. Integrated stress response of vertebrates is regulated by four eIF $2 \alpha$ kinases. Sci Rep. 2016;6(1):32886.

29. Sidrauski C, et al. The small molecule ISRIB reverses the effects of eIF2alpha phosphorylation on translation and stress granule assembly. Elife. 2015;4:e05033.

30. Özcan U, et al. Chemical chaperones reduce ER stress and restore glucose homeostasis in a mouse model of type 2 diabetes. Science. 2006;313(5790):1137-1140.

31. Ingrand S, et al. The oxindole/imidazole derivative $\mathrm{C} 16$ reduces in vivo brain $\mathrm{PKR}$ activation FEBS Lett. 2007;581(23):4473-4478.

32. Hull CM, Bevilacqua PC. Discriminating self and non-self by RNA: roles for RNA structure, misfolding, and modification in regulating the innate immune sensor PKR. Acc Chem Res. 
2016;49(6):1242-1249.

33. Nakamura T. dsRNA in immunometabolism. Oncotarget. 2015;6(24):19940-19941.

34. Weber F, et al. Double-stranded RNA is produced by positive-strand RNA viruses and DNA viruses but not in detectable amounts by negative-strand RNA viruses. J Virol. 2006;80(10):5059-5064.

35. Nakamura T, et al. Double-stranded RNAdependent protein kinase links pathogen sensing with stress and metabolic homeostasis. Cell. 2010;140(3):338-348.

36. Zhang Y, et al. The flavoheme reductase Ncb5or protects cells against endoplasmic reticulum stress-induced lipotoxicity. J Lipid Res. 2010;51(1):53-62.

37. Lorenzon-Ojea AR, et al. Stromal cell derived factor-2 (Sdf2): a novel protein expressed in mouse. Int J Biochem Cell Biol. 2014;53:262-270.

38. Garg B, et al. Signal sequence receptor 2 is required for survival of human melanoma cells as part of an unfolded protein response to endoplasmic reticulum stress. Mutagenesis. 2016;31(5):573-582.

39. Hori O, et al. Deletion of SERP1/RAMP4, a component of the endoplasmic reticulum (ER) translocation sites, leads to ER stress. Mol Cell Biol. 2006;26(11):4257-4267.

40. Ji DX, et al. Role of the transcriptional regulator SP140 in resistance to bacterial infections via repression of type I interferons [preprint]. https://doi.org/10.1101/2020.01.07.897553. Posted on bioRxiv January 8, 2020.

41. Sidrauski C, et al. Pharmacological dimerization and activation of the exchange factor eIF2B antagonizes the integrated stress response. Elife. 2015;4:e07314.

42. Pakos Zebrucka K, et al. The integrated stress response. EMBO reports. 2016;17(10):1374-1395.

43. Belton M, et al. Hypoxia and tissue destruction in pulmonary TB. Thorax. 2016;71(12):1145-1153.

44. Khan NA, et al. mTORC1 regulates mitochondrial integrated stress response and mitochondrial myopathy progression. Cell Metab. 2017;26(2):419-428.e5.

45. Gutierrez MG, et al. Autophagy is a defense mechanism inhibiting BCG and Mycobacterium tuberculosis survival in infected macrophages.
Cell. 2004;119(6):753-766.

46. Rodrigo CM, et al. Synthesis of rocaglamide hydroxamates and related compounds as eukaryotic translation inhibitors: synthetic and biological studies. J Med Chem. 2012;55(1):558-562.

47. Manier S, et al. Inhibiting the oncogenic translation program is an effective therapeutic strategy in multiple myeloma. Sci Transl Med. 2017;9(389):eaal2668.

48. Martinon F, Glimcher LH. Regulation of innate immunity by signaling pathways emerging from the endoplasmic reticulum. Curr Opin Immunol. 2011;23(1):35-40.

49. Valderrama C, et al. Listeria monocytogenes induces an interferon-enhanced activation of the integrated stress response that is detrimental for resolution of infection in mice. Eur J Immunol. 2017;47(5):830-840.

50. Yamashiro LH, et al. Interferon-independent STING signaling promotes resistance to HSV-1 in vivo. Nat Commun. 2020;11(1):3382.

51. Panne D, et al. An atomic model of the interferon-beta enhanceosome. Cell. 2007;129(6):1111-1123.

52. Wu H, et al. JNK protects Drosophila from oxidative stress by trancriptionally activating autophagy. Mech Dev. 2009;126(8-9):624-637.

53. Bunyard P, et al. Ribotoxic stress activates p38 and JNK kinases and modulates the antigenpresenting activity of dendritic cells. Mol Immunol. 2003;39(13):815-827.

54. Robertson KA, Ghazal P. Interferon control of the sterol metabolic network: bidirectional molecular circuitry-mediating host protection. Front Immunol. 2016;7:634.

55. Shibata N, et al. 25-Hydroxycholesterol activates the integrated stress response to reprogram transcription and translation in macrophages. J Biol Chem. 2013;288(50):35812-35823.

56. York AG, et al. Limiting cholesterol biosynthetic flux spontaneously engages type I IFN signaling. Cell. 2015;163(7):1716-1729.

57. Gold ES, et al. 25-Hydroxycholesterol acts as an amplifier of inflammatory signaling. Proc Natl Acad Sci U S A. 2014;111(29):10666-10671.

58. Gierahn TM, et al. Seq-Well: portable, low-cost RNA sequencing of single cells at high through- put. Nat Methods. 2017;14(4):395-398.

59. Desvignes L, et al. Dynamic roles of type I and type II IFNs in early infection with Mycobacterium tuberculosis. J Immunol. 2012;188(12):6205-6215.

60. Sidrauski C, et al. Pharmacological brake-release of mRNA translation enhances cognitive memory. Elife. 2013;2:e00498.

61. Hosoi T, et al. Unique pharmacological property of ISRIB in inhibition of A $\beta$-induced neuronal cell death. J Pharmacol Sci. 2016;131(4):292-295.

62. Williams MS, et al. A stress-responsive enhancer induces dynamic drug resistance in acute myeloid leukemia. J Clin Invest. 2020;130(3):1217-1232.

63. Chou A, et al. Inhibition of the integrated stress response reverses cognitive deficits after traumatic brain injury. Proc Natl Acad Sci U S A. 2017;114(31):E6420-E6426.

64. Sekine Y, et al. Stress responses. Mutations in a translation initiation factor identify the target of a memory-enhancing compound. Science. 2015;348(6238):1027-1030.

65. Nguyen HG, et al. Development of a stress response therapy targeting aggressive prostate cancer. Sci Transl Med. 2018;10(439):eaar2036.

66. Pan H, et al. Dual-promoter lentiviral system allows inducible expression of noxious proteins in macrophages. JImmunol Methods. 2008;329(1-2):31-44.

67. Gupta S, et al. Acceleration of tuberculosis treatment by adjunctive therapy with verapamil as an efflux inhibitor. Am J Respir Crit Care Med. 2013;188(5):600-607.

68. Ihms EA, et al. Diverse cavity types and evidence that mechanical action on the necrotic granuloma drives tuberculous cavitation. Am J Pathol. 2018;188(7):1666-1675.

69. Xu Z, et al. Computer-aided pulmonary image analysis in small animal models. Med Phys. 2015;42(7):3896-3910.

70. Dempster AP, et al. Maximum likelihood from incomplete data via the EM algorithm. JR Stat Soc Series B Stat Methodol. 1977;39(1):1-38.

71. Bhattacharya $B$, et al. Fine-tuning of macrophage activation using synthetic rocaglate derivatives. Sci Rep. 2016;6:24409. 